\title{
Systematic and variational truncation of the configuration space in the multiconfiguration time-dependent Hartree method: The MCTDH[n] hierarchy
}

Cite as: J. Chem. Phys. 152, 084101 (2020); https://doi.org/10.1063/1.5142459

Submitted: 13 December 2019 . Accepted: 28 January 2020 . Published Online: 24 February 2020

Niels Kristian Madsen (D), Mads Bøttger Hansen (D), Graham A. Worth (D), and Ove Christiansen (D)

\section{ARTICLES YOU MAY BE INTERESTED IN}

The multi-configurational time-dependent Hartree approach in optimized second quantization: Imaginary time propagation and particle number conservation

The Journal of Chemical Physics 152, 034101 (2020); https://doi.org/10.1063/1.5140984

The density matrix renormalization group in chemistry and molecular physics: Recent developments and new challenges

The Journal of Chemical Physics 152, 040903 (2020); https://doi.org/10.1063/1.5129672

MCSCF optimization revisited. II. Combined first- and second-order orbital optimization for large molecules

The Journal of Chemical Physics 152, 074102 (2020); https://doi.org/10.1063/1.5142241

Lock-in Amplifiers Find out more today

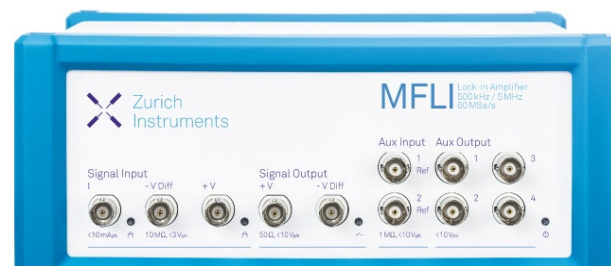

Zurich - instruments 


\title{
Systematic and variational truncation of the configuration space in the multiconfiguration time-dependent Hartree method: The MCTDH $[n]$ hierarchy
}

\author{
Cite as: J. Chem. Phys. 152, 084101 (2020); doi: 10.1063/1.5142459 \\ Submitted: 13 December 2019 - Accepted: 28 January 2020 • \\ Published Online: 24 February 2020
}

\begin{abstract}
Niels Kristian Madsen, ${ }^{1, a)}$ (D) Mads Bøttger Hansen, ${ }^{1}$ Graham A. Worth, ${ }^{2}$

\author{
AFFILIATIONS \\ 'Department of Chemistry, University of Aarhus, Langelandsgade 140, DK-8000 Aarhus C, Denmark \\ ${ }^{2}$ Department of Chemistry, University College London, 20, Gordon St., WC1H OAJ London, United Kingdom
} and Ove Christiansen

a) Author to whom correspondence should be addressed: nielskm@chem.au.dk

\begin{abstract}
The multiconfiguration time-dependent Hartree (MCTDH) method is a powerful method for solving the time-dependent Schrödinger equation in quantum molecular dynamics. It is, however, hampered by the so-called curse of dimensionality which results in exponential scaling with respect to the number of degrees of freedom in the system and, thus, limits its applicability to small- and medium-sized molecules. To avoid this scaling, we derive equations of motion for a series of truncated MCTDH methods using a many-mode second-quantization formulation where the configuration space is restricted based on mode-combination levels as also done in the vibrational configuration interaction and vibrational coupled cluster methods for solving the time-independent Schrödinger equation. The full MCTDH wave function is invariant with respect to the choice of constraint (or gauge) operators, but restricting the configuration space removes this invariance. We, thus, analyze the remaining redundancies and derive equations for variationally optimizing the non-redundant matrix elements of the constraint operators. As an alternative, we also present a constraint that keeps the density matrices block diagonal during the propagation and the two choices are compared. Example calculations are performed on formyl fluoride and a series of high-dimensional Henon-Heiles potentials. The results show that the MCTDH $[n]$ methods can be applied to large systems and that an optimal choice of constraint operators is key to obtaining the correct physical behavior of the wave function.
\end{abstract}

Published under license by AIP Publishing. https://doi.org/10.1063/1.5142459

\section{INTRODUCTION}

Quantum molecular dynamics simulations are important tools for understanding time-dependent processes in molecules such as chemical reactions, non-adiabatic transitions, and systems with time-dependent Hamiltonians. For few-dimensional systems, the time-dependent Schrödinger equation (TDSE) can be solved very efficiently using standard numerical methods. ${ }^{1}$ However, applying these methods to slightly larger systems (more than four atoms) quickly becomes impossible due to the inherent exponential scaling with respect to the system size known as the curse of dimensionality. Over the years, numerous methods with different wave-function parameterizations and propagation schemes have been developed for extending the range of applicability of quantum molecular dynamics.

One of the well-established methods is multiconfiguration time-dependent Hartree (MCTDH), ${ }^{2,3}$ which provides a versatile and efficient framework for time-dependent wave-packet propagation. It applies the time-dependent variational principle (TDVP) to a multi-configurational Ansatz expanded in time-dependent Hartree products and is thus a compact way of representing the exact wave function on a basis that adapts itself to the evolving wave packet. By varying the number of time-dependent functions used to describe each degree of freedom (often called single-particle 
functions (SPFs), but we denote them as time-dependent modals in line with our previous work ${ }^{4}$ ), the MCTDH method spans between the exact wave function and time-dependent Hartree (TDH) in the limit of a single time-dependent Hartree product. The TDH method is an important approximation in its own right and has recently been reformulated in second quantization (SQ) and an exponential parameterization, which makes it applicable to study systems with more than $10^{5}$ degrees of freedom. ${ }^{4}$ However, the accuracy of TDH is limited and a multi-configurational wave function is required for obtaining physically correct results for many systems of interest. ${ }^{3,5}$ Compared to the exact method, MCTDH allows converged results for much larger systems to be obtained. The computational scaling is, however, still exponential with respect to the number of degrees of freedom. Several methods have been developed to reduce the computational cost and scaling of MCTDH such as multilayer MCTDH (ML-MCTDH) ${ }^{6-9}$ and alternative formulations based on various forms of time-dependent Gaussian bases such as Gaussian MCTDH (G-MCTDH), ${ }^{10,11}$ variational multi-configurational Gaussian (vMCG), ${ }^{12,13}$ and coupled coherent states (CCS). ${ }^{14,15}$ These are in turn related to multiple spawning methods (see Ref. 16 for a recent review).

In this paper, we take a different approach to mitigating the exponential scaling of MCTDH. Following previous ideas, ${ }^{17-20}$ we seek to reduce the number of configurations included in the MCTDH wave function. We propose a systematic way by formulating a hierarchy of $\mathrm{MCTDH}[n]$ methods, which include up to $n$-mode excitations with respect to a selected reference configuration. This choice of configurations is in line with our vibrational configuration interaction (VCI) ${ }^{21,22}$ and vibrational coupled cluster (VCC) ${ }^{23-25}$ methods for solving the time-independent Schrödinger equation. Thus, $\mathrm{MCTDH}[n]$ converges toward full MCTDH in the limit where $n$ equals the number of degrees of freedom in the system, $M$. The equations of motion (EOMs) for MCTDH and $\operatorname{MCTDH}[n]$ are derived using a SQ formalism ${ }^{21}$ to keep the formulation in line with our previous work on time-independent theory, $\mathrm{TDH},{ }^{4}$ and time-dependent vibrational coupled cluster (TDVCC). ${ }^{2}$ In addition, once the SQ is in place, SQ provides a short derivation compared to first quantization with natural introduction of key quantities for both $\mathrm{MCTDH}$ and $\mathrm{MCTDH}[n]$ bringing also some resemblance to SQ formulations in electronic-structure theory. We note that SQ formulations have previously been introduced in the context of (ML-)MCTDH for systems of indistinguishable particles. ${ }^{9,27,28,30,31}$ Our SQ treats distinguishable degrees of freedom (modes) and, thus, differs in the sense that a set of elementary operators is introduced for each mode.

The full MCTDH wave function is invariant with respect to unitary transformations of the time-dependent modals. This redundancy is removed by a choice of Hermitian constraint (or gauge) operators, $\tilde{g}_{u^{m} v^{m}}^{m}=i\left\langle\tilde{\phi}_{u^{m}}^{m}\left(q_{m}, t\right) \mid \dot{\tilde{\phi}}_{v^{m}}^{m}\left(q_{m}, t\right)\right\rangle$ (with $\tilde{\phi}_{u^{m}}^{m}$ being the $u^{m}$ 'th time-dependent modal in mode $m$ ), which are often just set to zero. Using only a selected set of configurations, however, removes parts of this redundancy and makes the choice of constraint operators affect the accuracy of the wave function. In previous studies ${ }^{17-20}$ the simple choice of $g=0$ has been employed in the EOMs combined with a transformation to natural modals (orbitals) after each propagation step in order to keep the importance of the missing configurations small. This ad hoc procedure is physically motivated, but each transformation changes the wave function if the configuration space is truncated. That is, in general more configurations are needed to express the same wave function in the transformed basis and if these are neglected, the transformation becomes inexact. Thus, the time evolution of the wave packet becomes dependent on the frequency of the basis transformations, i.e., the step size of the integrator. In this work, we derive EOMs for MCTDH $[n]$ where the constraint operators are variationally optimized in line with previous work on MCTDH for systems of identical fermions ${ }^{31-33}$ and bosons. ${ }^{34}$ It is shown that the non-redundant matrix elements of the constraint operators, which keep the $\mathrm{MCTDH}[n]$ wave function variationally optimal can be obtained by solving a set of linear equations. These equations correspond to a linear least squares (LLS) minimization of the time derivative of the $(n+1)$-excited configurations, and thus, the effect of adding the next level of excitations is minimized. We furthermore discuss an alternative constraint, which keeps the one-mode density matrices block diagonal and, thus, makes the reference configuration a product of natural modals. This choice keeps the density matrices block diagonal by construction of the EOMs unlike the ad hoc approach described above. The fully variational and the density-matrix constraints are compared to the simple choice of $g=0$ for different $\operatorname{MCTDH}[n]$ methods in a series of test calculations.

The remainder of the paper is structured as follows. Section II presents the general terminology and theoretical foundation as well as the SQ formulation used in the derivation of the EOMs. In Sec. III, the MCTDH EOMs are derived in SQ and the MCTDH $[n]$ methods are presented in Sec. IV. Section V describes the implementation, while Sec. VI presents the numerical results. Finally, Sec. VII presents a summary and future outlook.

\section{GENERAL THEORY}

Considering a system with $M$ degrees of freedom (modes) with coordinates $\mathbf{q}=\left\{q_{1}, q_{2}, \ldots, q_{M}\right\}$, the exact time-dependent wave function can be written as a linear combination of Hartree products, $\Phi_{\mathbf{r}}$,

$$
\bar{\Psi}(\mathbf{q}, t)=\sum_{\mathbf{r}} B_{\mathbf{r}}(t) \Phi_{\mathbf{r}}(\mathbf{q}) .
$$

The Hartree product is a product of one-dimensional functions denoted as modals,

$$
\Phi_{\mathbf{r}}=\prod_{m=1}^{M} \phi_{r^{m}}^{m}\left(q_{m}\right),
$$

where $r^{m}=0,1, \ldots, N^{m}-1$ for each mode. The wave function in Eq. (1) is expanded on the basis of time-independent Hartree products. However, it is also possible to use a time-dependent Hartreeproduct basis $\left\{\tilde{\Phi}_{\mathbf{r}}(\mathbf{q}, t)\right\}$. This allows for a more compact form of the wave function, which is the foundation for the MCTDH method introduced in Sec. III.

In the following sections, all wave functions and operators will be represented using the SQ formulation presented in Sec. II A.

\section{A. Second-quantization formulation of many-mode dynamics}

Following the SQ formulation of Ref. 21, vibrational wave functions and operators are represented by creation $a_{r^{m}}^{m \dagger}$ and 
annihilation $a_{r^{m}}^{m}$ operators, which create or annihilate occupation in modal $r^{m}$ of mode $m$. These elementary operators satisfy the commutator relations,

$$
\begin{aligned}
{\left[a_{r^{m}}^{m}, a_{s^{m^{\prime}}}^{m^{\prime} \dagger}\right] } & =\delta_{m m^{\prime}} \delta_{r^{m} s^{m^{\prime}}}, \\
{\left[a_{r^{m}}^{m}, a_{s^{m^{\prime}}}^{m^{\prime}}\right] } & =0, \\
{\left[a_{r^{m}}^{m \dagger}, a_{s^{m^{\prime}}}^{m^{\prime} \dagger}\right] } & =0 .
\end{aligned}
$$

The state with no occupation is denoted as the vacuum state $|\mathrm{vac}\rangle$, which satisfies $\langle\operatorname{vac} \mid \mathrm{vac}\rangle=1$ and the killer condition,

$$
a_{r^{m}}^{m}|\operatorname{vac}\rangle=0
$$

A time-dependent Hartree product can be written as

$$
\left|\tilde{\Phi}_{\mathbf{r}}\right\rangle=\prod_{m=1}^{M} \tilde{a}_{r^{m}}^{m \dagger}|\mathrm{vac}\rangle
$$

where the $\tilde{a}_{r^{m}}^{m \dagger}$ operators create time-dependent modals. If the timedependent creation operators are related to those of the timeindependent basis by a unitary transformation, the $\tilde{a}_{r^{m}}^{m \dagger}$ and $\tilde{a}_{s^{m}}^{m}$ operators also satisfy Eq. (3) (we refer to Ref. 4 for further discussion of time-dependent creation and annihilation operators). In order to manipulate wave functions, we introduce the number-conserving shift operator,

$$
\tilde{E}_{r^{m} s^{m}}^{m} \equiv \tilde{a}_{r^{m}}^{m \dagger} \tilde{a}_{s^{m}}^{m},
$$

which shifts occupation from modal $s^{m}$ to $r^{m}$ in mode $m$.

\section{Operators in second quantization}

A general Hermitian operator of the form $O=O_{0}+\sum_{m} O^{m}+\cdots$ can be written in SQ as

$$
\begin{aligned}
O= & O_{0}+\sum_{m} \sum_{r^{m} s^{m}} O_{r^{m} s^{m}}^{m} E_{r^{m} s^{m}}^{m} \\
& +\sum_{m_{1}<m_{2}} \sum_{r^{m_{1}} r^{m_{2}} s^{m_{1}} s^{m_{2}}} O_{r^{m_{1}} r^{m_{2}} s^{m_{1}} s^{m_{2}}}^{m_{2}} E_{r^{m_{1}} s^{m_{1}}}^{m_{1}} E_{r^{m_{2}} s^{m_{2}}}^{m_{2}}+\ldots,
\end{aligned}
$$

with integrals

$$
O_{r^{m_{1}} r^{m_{2}} \ldots m_{n} m_{n} s^{m_{1}} s^{m_{2}} \ldots s^{m_{n}}}^{m_{1} m_{2}} \equiv\left\langle r^{m_{1}} r^{m_{2}} \ldots r^{m_{n}}\left|O^{m_{1} m_{2} \ldots m_{n}}\right| s^{m_{1}} s^{m_{2}} \ldots s^{m_{n}}\right\rangle .
$$

The exact vibrational Hamiltonian operator can be represented by such an expansion, but since the exact potential-energy surface (PES) is generally not known, we often choose to represent the Hamiltonian in the computationally favorable sum-of-products (SOP) form, ${ }^{3,23,35}$

$$
H=\sum_{t} c_{t} \prod_{m \in \mathbf{m}_{t}} h^{m t},
$$

where $\mathbf{m}_{t}$ is a mode combination (MC), i.e., the set of modes that are operated on by term $t$. The one-mode operators can be written both in terms of the time-independent and the time-dependent basis as

$$
h^{m t}=\sum_{r^{m} s^{m}} h_{r^{m} s^{m}}^{m t} a_{r^{m}}^{m \dagger} a_{s^{m}}^{m}=\sum_{r^{m} s^{m}} \tilde{h}_{r^{m} s^{m}}^{m t}(t) \tilde{a}_{r^{m}}^{m \dagger}(t) \tilde{a}_{s^{m}}^{m}(t) .
$$

\section{MCTDH IN SECOND QUANTIZATION}

We now introduce the MCTDH method, which is a very efficient method for representing the exact time-dependent wave function [Eq. (1)] in a compact way by employing a variationally optimized, time-dependent modal basis for each mode.

\section{A. Definitions}

MCTDH has previously been derived in first quantization (FQ). ${ }^{2,3}$ We present here a new derivation based on a manymode SQ formulation $^{21}$ in line with our previous work on timeindependent vibrational-structure theory and $\mathrm{TDH}^{4}$. Note that our SQ formulation is fundamentally different from harmonicoscillator ladder operators applied to anharmonic vibrational problems. ${ }^{36}$

In MCTDH, the modal space of each vibrational mode is divided into an active space of size $n^{m}$ with indices $u^{m}, v^{m}$, and $w^{m}$ and a secondary space of size $\left(N^{m}-n^{m}\right)$ with indices $x^{m}$ and $y^{m}$. The indices $r^{m}$ and $s^{m}$ denote modals of unspecified occupancy and we use greek letters $\alpha^{m}$ and $\beta^{m}$ to denote modals belonging to the underlying primitive basis. The MCTDH wave function is then written as

$$
|\bar{\Psi}\rangle=\sum_{u^{1}=1}^{n^{1}} \ldots \sum_{u^{M}=1}^{n^{M}} C_{u^{1} \ldots u^{M}}(t) \prod_{m=1}^{M} \tilde{a}_{u^{m}}^{m \dagger}(t)|\mathrm{vac}\rangle=\sum_{\mathbf{u}} C_{\mathbf{u}}\left|\tilde{\Phi}_{\mathbf{u}}\right\rangle,
$$

where the $\tilde{a}_{u^{m}}^{m \dagger}(t)$ operators create the time-dependent modals. The time-dependent modals are assumed to be orthonormal at time $t=0$, i.e., the standard commutator relations [Eq. (3)] apply. In order to conserve orthonormality between the active-space modals $\left[\frac{d}{d t}\left[\tilde{a}_{u^{m}}^{m}(t), \tilde{a}_{v^{m^{\prime}}}^{m^{\prime}}(t)\right]=0\right]$, the following commutator relation must be satisfied:

$$
\left[\tilde{a}_{u^{m}}^{m}(t), \dot{\tilde{a}}_{v^{m}}^{m \dagger}(t)\right]=-i \tilde{g}_{u^{m}}^{m} v^{m}(t),
$$

where

$$
g^{m}=\sum_{u^{m} v^{m}} \tilde{g}_{u^{m}}^{m} v^{m} \tilde{E}_{\mathcal{u}^{m}}^{m} v^{m}
$$

is a Hermitian (but otherwise arbitrary) one-mode operator ${ }^{3}$ denoted in the following as the constraint operator for mode $m$. Note that the FQ analog to (11) is $\left\langle\tilde{\phi}_{u^{m}}^{m}\left(q_{m}, t\right) \mid \dot{\tilde{\phi}}_{v^{m}}^{m}\left(q_{m}, t\right)\right\rangle=-i \tilde{g}_{u^{m}}^{m} v^{m}(t)$, which is a well-known expression in the MCTDH theory. ${ }^{3}$ This gauge freedom is a result of the fact that the active spaces can be rotated without changing the total wave function. If the configuration space is truncated, however, some elements of the $g^{m}$ matrices become non-redundant. This issue will be addressed in Sec. IV.

Before deriving the MCTDH EOMs, we introduce the following definitions. The notation is a necessary hybrid of what is standard in the MCTDH community ${ }^{3}$ with our formulation $\left(\mathbf{m}, \mathbf{u}^{\mathbf{m}}\right)$ used in previous VCC, VCI, and TDVCC ${ }^{26}$ works.

Single-hole functions:

$$
\left|\bar{\Psi}_{u^{m}}^{-m}\right\rangle=\tilde{a}_{u^{m}}^{m}|\bar{\Psi}\rangle=\bar{R}_{u^{m}}^{-m \dagger}|\mathrm{vac}\rangle
$$

Single-hole coefficients:

$$
C_{\mathbf{u}_{v^{m}}^{m}}=C_{u^{1} \ldots u^{m-1} v^{m} u^{m+1} \ldots u^{M}}(t) .
$$


Single-hole Hartree products:

$$
\left|\tilde{\Phi}_{\mathbf{u}}^{-m}\right\rangle=\tilde{a}_{u^{m}}^{m}\left|\tilde{\Phi}_{\mathbf{u}}\right\rangle=\tilde{A}_{\mathbf{u}}^{-m \dagger}|\mathrm{vac}\rangle
$$

Restricted summation:

$$
\sum_{\mathbf{r}}^{-m}=\sum_{r^{1}} \cdots \sum_{r^{m-1}} \sum_{r^{m+1}} \cdots \sum_{r^{M}} .
$$

Density matrices:

$$
\tilde{D}_{u^{m} v^{m}}^{m}=\left\langle\bar{\Psi}\left|\tilde{E}_{u^{m}}^{m} v^{m}\right| \bar{\Psi}\right\rangle=\left\langle\operatorname{vac}\left|\bar{R}_{\mathcal{u}^{m}}^{-m} \bar{R}_{v^{m}}^{-m \dagger}\right| \operatorname{vac}\right\rangle .
$$

Mean-field matrix elements:

$$
\tilde{F}_{u^{m} r^{m}}^{m}=\left\langle\bar{\Psi}\left|\tilde{a}_{u^{m}}^{m \dagger}\left[\tilde{a}_{r^{m}}^{m}, H\right]\right| \bar{\Psi}\right\rangle .
$$

We note that the SQ definition of the mean-field matrix elements is analogous to the generalized Fock matrix used in electronic-structure Hartree-Fock (HF) and multi-configurational self-consistent field (MCSCF) theory.

\section{B. SQ derivation of the MCTDH EOMs}

The EOMs are derived using the Dirac-Frenkel TDVP, 2,

$$
\left\langle\delta \bar{\Psi}\left|\left(H-i \frac{\partial}{\partial t}\right)\right| \bar{\Psi}\right\rangle=0
$$

using the variation,

$$
|\delta \bar{\Psi}\rangle=\sum_{\mathbf{u}} \delta C_{\mathbf{u}}\left|\tilde{\Phi}_{\mathbf{u}}\right\rangle+\sum_{m=1}^{M} \sum_{u^{m}} \delta \tilde{a}_{u^{m}}^{m \dagger}\left|\bar{\Psi}_{u^{m}}^{-m}\right\rangle,
$$

where $\delta \tilde{a}_{u^{m}}^{m \dagger}$ is a linear variation of the creation operator for modal $u^{m}$ of mode $m$. We also introduce the time derivative,

$$
|\dot{\bar{\Psi}}\rangle=\sum_{\mathbf{u}} \dot{C}_{\mathbf{u}}\left|\tilde{\Phi}_{\mathbf{u}}\right\rangle+\sum_{m=1}^{M} \sum_{u^{m}} \dot{\tilde{a}}_{u^{m}}^{m \dagger}\left|\bar{\Psi}_{u^{m}}^{-m}\right\rangle
$$

Varying the $C_{\mathbf{u}}$ coefficients leads to

$$
\left\langle\tilde{\Phi}_{\mathbf{u}}|H| \bar{\Psi}\right\rangle-i\left\langle\tilde{\Phi}_{\mathbf{u}} \mid \dot{\bar{\Psi}}\right\rangle=0
$$

which results in the EOM of the configuration-space coefficients,

$$
\begin{aligned}
i \dot{C}_{\mathbf{u}} & =\left\langle\tilde{\Phi}_{\mathbf{u}}|H| \bar{\Psi}\right\rangle-i \sum_{m=1}^{M} \sum_{v^{m}}\left\langle\tilde{\Phi}_{\mathbf{u}}\left|\dot{\tilde{a}}_{v^{m}}^{m \dagger}\right| \bar{\Psi}_{v^{m}}^{-m}\right\rangle \\
& =\left\langle\tilde{\Phi}_{\mathbf{u}}|H| \bar{\Psi}\right\rangle-i \sum_{m=1}^{M} \sum_{v^{m}}\left\langle\tilde{\Phi}_{\mathbf{u}}^{-m}\left|\left[\tilde{a}_{u^{m}}^{m}, \dot{\tilde{a}}_{v^{m}}^{m \dagger}\right]\right| \bar{\Psi}_{v^{m}}^{-m}\right\rangle \\
& =\sum_{\mathbf{v}}\left\langle\tilde{\Phi}_{\mathbf{u}}|H| \tilde{\Phi}_{\mathbf{v}}\right\rangle C_{\mathbf{v}}-\sum_{m=1}^{M} \sum_{v^{m}} \tilde{g}_{u^{m}}^{m} v^{m} C_{\mathbf{u}_{v^{m}}^{m}} \\
& =\left\langle\tilde{\Phi}_{\mathbf{u}}|(H-g)| \bar{\Psi}\right\rangle,
\end{aligned}
$$

using $\left\langle\tilde{\Phi}_{\mathbf{u}}\right|=\left\langle\tilde{\Phi}_{\mathbf{u}}^{-m}\right| \tilde{a}_{u^{m}}^{m}$ from (15) as well as Eq. (4). In the last equality, we have introduced $g=\sum_{m} g^{m}$. Independent variation of the creation operators corresponding to the active modals results in

$$
\left\langle\bar{\Psi}_{u^{m}}^{-m}\left|\delta \tilde{a}_{u^{m}}^{m} H\right| \bar{\Psi}\right\rangle-i\left\langle\bar{\Psi}_{u^{m}}^{-m}\left|\delta \tilde{a}_{u^{m}}^{m}\right| \dot{\bar{\Psi}}\right\rangle=0 .
$$

Inserting Eqs. (21) and (23) leads to

$$
\begin{aligned}
\left\langle\bar{\Psi}_{u^{m}}^{-m}\left|\delta \tilde{a}_{u^{m}}^{m}\left(I-\sum_{\mathbf{w}}\left|\tilde{\Phi}_{\mathbf{w}}\right\rangle\left\langle\tilde{\Phi}_{\mathbf{w}}\right|\right) H\right| \bar{\Psi}\right\rangle= & i \sum_{m^{\prime}} \sum_{v^{m^{\prime}}}\left\langle\bar{\Psi}_{u^{m}}^{-m}\left|\delta \tilde{a}_{u^{m}}^{m} \dot{\tilde{a}}_{v^{m^{\prime}}}^{m^{\prime} \dagger}\right| \bar{\Psi}_{v^{m^{\prime}}}^{-m^{\prime}}\right\rangle \\
& -\left\langle\bar{\Psi}_{u^{m}}^{-m}\left|\delta \tilde{a}_{u^{m}}^{m} g\right| \bar{\Psi}\right\rangle .
\end{aligned}
$$

We now introduce the resolution of the identity written as a purely active-space part, a single-excited (to secondary space) part, and higher excitation levels,

$$
I=\sum_{\mathbf{u}}\left|\tilde{\Phi}_{\mathbf{u}}\right\rangle\left\langle\tilde{\Phi}_{\mathbf{u}}\left|+\sum_{m} \sum_{\mathbf{u}}^{-m} \sum_{x^{m}} \tilde{a}_{x^{m}}^{m \dagger}\right| \tilde{\Phi}_{\mathbf{u}}^{-m}\right\rangle\left\langle\tilde{\Phi}_{\mathbf{u}}^{-m}\right| \tilde{a}_{x^{m}}^{m}+\ldots
$$

This enables us to write the left-hand side (LHS) of (25) as

$$
\begin{aligned}
& \left\langle\bar{\Psi}_{u^{m}}^{-m}\left|\delta \tilde{a}_{u^{m}}^{m}\left(I-\sum_{\mathbf{w}}\left|\tilde{\Phi}_{\mathbf{w}}\right\rangle\left\langle\tilde{\Phi}_{\mathbf{w}}\right|\right) H\right| \bar{\Psi}\right\rangle \\
& =\sum_{x^{m}} \sum_{\mathbf{w}}^{-m}\left\langle\bar{\Psi}_{u^{m}}^{-m}\left|\delta \tilde{a}_{u^{m}}^{m} \tilde{a}_{x^{m}}^{m \dagger}\right| \tilde{\Phi}_{\mathbf{w}}^{-m}\right\rangle\left\langle\tilde{\Phi}_{\mathbf{w}}^{-m}\left|\tilde{a}_{x^{m}}^{m} H\right| \bar{\Psi}\right\rangle \\
& =\sum_{x^{m}}\left[\delta \tilde{a}_{u^{m}}^{m}, \tilde{a}_{x^{m}}^{m \dagger}\right] \sum_{\mathbf{w}}^{-m}\left\langle\bar{\Psi}_{u^{m}}^{-m} \mid \tilde{\Phi}_{\mathbf{w}}^{-m}\right\rangle\left\langle\tilde{\Phi}_{\mathbf{w}}^{-m}\left|\left[\tilde{a}_{x^{m}}^{m}, H\right]\right| \bar{\Psi}\right\rangle \\
& =\sum_{x^{m}}\left[\delta \tilde{a}_{u^{m}}^{m}, \tilde{a}_{x^{m}}^{m \dagger}\right]\left\langle\tilde{\Psi}_{u^{m}}^{-m}\left|\left[\tilde{a}_{x^{m}}^{m}, H\right]\right| \bar{\Psi}\right\rangle \\
& =\sum_{x^{m}}\left[\delta \tilde{a}_{u^{m}}^{m}, \tilde{a}_{x^{m}}^{m \dagger}\right] \tilde{F}_{u^{m} x^{m}}^{m} .
\end{aligned}
$$

In the first step, we note that on inserting Eq. (26) only terms that are one-mode excited in mode $m$ can contribute due to the bra. In the second step, we have used $\delta \tilde{a}_{u^{m}}^{m} \tilde{a}_{x^{m}}^{m \dagger}=\tilde{a}_{x^{m}}^{m \dagger} \delta \tilde{a}_{u^{m}}^{m}+\left[\delta \tilde{a}_{u^{m}}^{m}, \tilde{a}_{x^{m}}^{m \dagger}\right]$ and noted that the first term is zero when applied to the bra and the commutator is a scalar which can be moved outside the bra-ket. The right-hand side (RHS) of Eq. (25) is reduced to

$$
\begin{aligned}
i \sum_{m^{\prime}} \sum_{v^{m^{\prime}}}\left\langle\bar{\Psi}_{u^{m}}^{-m}\left|\delta \tilde{a}_{u^{m}}^{m} \dot{\tilde{a}}_{v^{m^{\prime}}}^{m^{\prime} \dagger}\right| \bar{\Psi}_{v^{m^{\prime}}}^{-m^{\prime}}\right\rangle-\left\langle\bar{\Psi}_{u^{m}}^{-m}\left|\delta \tilde{a}_{u^{m}}^{m} g\right| \bar{\Psi}\right\rangle \\
=\left\langle\operatorname{vac}\left|\bar{R}_{u^{m}}^{-m} \delta \tilde{a}_{u^{m}}^{m} \sum_{m^{\prime}} \sum_{v^{m^{\prime}}}\left(i \dot{\tilde{a}}_{v^{m^{\prime}}}^{m^{\prime} \dagger}-\sum_{w^{m^{\prime}}} \tilde{g}_{w^{m^{\prime}}}^{m^{\prime}} v^{m^{\prime}} \tilde{a}_{w^{m^{\prime}}}^{m^{\prime} \dagger}\right) \bar{R}_{v^{m^{\prime}}}^{-m^{\prime} \dagger}\right| \mathrm{vac}\right\rangle \\
=i \sum_{v^{m}}\left[\delta \tilde{a}_{u^{m}}^{m}, \dot{\tilde{a}}_{v^{m}}^{m \dagger}\right]\left\langle\operatorname{vac}\left|\bar{R}_{u^{m}}^{-m} \bar{R}_{v^{m}}^{-m \dagger}\right| \mathrm{vac}\right\rangle \\
\quad-\sum_{v^{m} w^{m}} \tilde{g}_{w^{m}}^{m} v^{m}\left[\delta \tilde{a}_{u^{m}}^{m}, \tilde{a}_{w^{m}}^{m \dagger}\right]\left\langle\operatorname{vac}\left|\bar{R}_{u^{m}}^{-m} \bar{R}_{v^{m}}^{-m \dagger}\right| \mathrm{vac}\right\rangle \\
=i \sum_{v^{m}} \tilde{D}_{u^{m}}^{m} v^{m}\left[\delta \tilde{a}_{u^{m}}^{m}, \dot{\tilde{a}}_{v^{m}}^{m \dagger}\right]-\sum_{v^{m} w^{m}} \tilde{D}_{u^{m} v^{m}}^{m} \tilde{g}_{w^{m} v^{m}}^{m}\left[\delta \tilde{a}_{u^{m}}^{m}, \tilde{a}_{w^{m}}^{m \dagger}\right],
\end{aligned}
$$

where we have used (3) several times. In the second equality, we have used the fact that the $m^{\prime} \neq m$ terms do not contribute since they contain factors that for mode $m^{\prime}$ correspond to

$$
\begin{aligned}
& \left\langle\operatorname{vac}\left|\tilde{a}_{u^{m^{\prime}}}^{m^{\prime}}\left(i \dot{\tilde{a}}_{v^{m^{\prime}}}^{m^{\prime} \dagger}-\sum_{w^{m^{\prime}}} \tilde{g}_{w^{m^{\prime}} v^{m^{\prime}}}^{m^{\prime}} \tilde{a}_{w^{m^{\prime}}}^{m^{\prime} \dagger}\right)\right| \mathrm{vac}\right\rangle \\
& =i\left[\tilde{a}_{u^{m^{\prime}}}^{m^{\prime}}, \dot{\tilde{a}}_{v^{m^{\prime}}}^{m^{\prime} \dagger}\right]-\sum_{w^{m^{\prime}}} \tilde{g}_{w^{m^{\prime}}}^{m^{\prime}} v^{m^{\prime}}\left[\tilde{a}_{u^{m^{\prime}}}^{m^{\prime}}, \tilde{a}_{w^{m^{\prime}}}^{m^{\prime} \dagger}\right] \\
& =\tilde{g}_{u^{m^{\prime}}}^{m^{\prime}} v^{m^{\prime}}-\sum_{w^{m^{\prime}}} \tilde{g}_{w^{m^{\prime}}}^{m^{\prime}} v^{m^{\prime}} \delta_{u^{m^{\prime}} w^{m^{\prime}}}=0 .
\end{aligned}
$$


Altogether, the EOM for the time-dependent modals is

$$
\begin{aligned}
i \sum_{v^{m}} \tilde{D}_{u^{m} v^{m}}^{m}\left[\delta \tilde{a}_{u^{m}}^{m}, \dot{\tilde{a}}_{v^{m}}^{m \dagger}\right]= & \sum_{x^{m}} \tilde{F}_{u^{m} x^{m}}^{m}\left[\delta \tilde{a}_{u^{m}}^{m}, \tilde{a}_{x^{m}}^{m \dagger}\right] \\
& +\sum_{v^{m} w^{m}} \tilde{D}_{u^{m} v^{m}}^{m} \tilde{g}_{w^{m}}^{m} v^{m}\left[\delta \tilde{a}_{u^{m}}^{m}, \tilde{a}_{w^{m}}^{m \dagger}\right] .
\end{aligned}
$$

\section{Linear parameterization of the time-dependent modals}

Equation (30) is valid for any parameterization of the timedependent modals (e.g., linear or exponential as discussed in Ref. 4). We choose a linear parameterization in terms of an orthonormal, time-independent primitive basis represented by the $a_{\alpha^{m}}^{m \dagger}$ and $a_{\beta^{m}}^{m}$ operators,

$$
\begin{aligned}
& \tilde{a}_{r^{m}}^{m \dagger}(t)=\sum_{\alpha^{m}} a_{\alpha^{m}}^{m \dagger} U_{\alpha^{m} r^{m}}^{m}(t), \\
& \tilde{a}_{s^{m}}^{m}(t)=\sum_{\beta^{m}} a_{\beta^{m}}^{m} U_{\beta^{m} s^{m}}^{m *}(t) .
\end{aligned}
$$

Inserting these into (30) results in expressions such as $\left[\delta \tilde{a}_{u^{m}}^{m}, \dot{\tilde{a}}_{v^{m}}^{m \dagger}\right]=\sum_{\alpha^{m} \beta^{m}} \delta U_{\alpha^{m} u^{m}}^{m *}\left[a_{\alpha^{m}}^{m}, a_{\beta^{m}}^{m \dagger}\right] \dot{U}_{\beta^{m} v^{m}}^{m}=\sum_{\alpha^{m}} \delta U_{\alpha^{m} u^{m}}^{m *} \dot{U}_{\alpha^{m} v^{m}}^{m}$ in all terms. Assuming arbitrary variations gets rid of the $\delta U_{\alpha^{m} u^{m}}^{m *}$ elements, resulting in

$$
i \dot{U}_{\alpha^{m} u^{m}}^{m}=\sum_{v^{m} x^{m}}\left[\left(\tilde{\mathbf{D}}^{m}\right)^{-1}\right]_{u^{m} v^{m}} \tilde{F}_{v^{m} x^{m}}^{m} U_{\alpha^{m} x^{m}}^{m}+\sum_{v^{m}} U_{\alpha^{m}}^{m} v^{m} \tilde{g}_{v^{m} u^{m}}^{m} .
$$

Note that the variations are in principle arbitrary, but the definition of the $g^{m}$ operators ensures that the $U^{m}$ matrices stay unitary at all times (disregarding numerical errors introduced by inexact propagation). The density matrices may become singular due to some active modals having zero weight in the wave function and, thus, the matrix inversion needs to be regularized as discussed in Refs. 3 and 38 . We note that a propagation scheme which avoids the densitymatrix inversion completely has been developed, ${ }^{39,40}$ but in this work, we apply standard numerical integrators and, thus, regularization is required. The $x^{m}$ summation requires the set of secondary modals which is only defined as the orthogonal complement to the active space. To avoid constructing the full orthogonal complement explicitly, we choose to convert this to an expression involving only the active-space modals,

$$
\begin{aligned}
\sum_{\chi^{m}} \tilde{F}_{v^{m} x^{m}}^{m} U_{\alpha^{m} x^{m}}^{m} & =\sum_{r^{m}} \tilde{F}_{v^{m} r^{m}}^{m} U_{\alpha^{m} r^{m}}^{m}-\sum_{u^{m}} \tilde{F}_{v^{m} u^{m}}^{m} U_{\alpha^{m} u^{m}}^{m} \\
& =\sum_{\beta^{m}}\left(\delta_{\alpha^{m} \beta^{m}}-\tilde{P}_{\alpha^{m} \beta^{m}}^{m}\right) \check{F}_{v^{m} \beta^{m}}^{m},
\end{aligned}
$$

where $\check{F}_{v^{m} \beta^{m}}^{m}=\sum_{r^{m}} \tilde{F}_{v^{m} r^{m}}^{m} U_{\beta^{m} r^{m}}^{m}$ [see (37) for the expression involving only active-space modals] and $\tilde{P}_{\alpha^{m} \beta^{m}}^{m}=\sum_{u^{m}} U_{\alpha^{m} u^{m}}^{m} U_{\beta^{m} u^{m}}^{m *}$ is a matrix element of the one-mode projector for mode $m$. This results in the final EOM,

$$
\begin{aligned}
i \dot{U}_{\alpha^{m} u^{m}}^{m}= & \sum_{\beta^{m}}\left(\delta_{\alpha^{m} \beta^{m}}-\tilde{P}_{\alpha^{m} \beta^{m}}^{m}\right) \sum_{v^{m}}\left[\left(\tilde{\mathbf{D}}^{m}\right)^{-1}\right]_{u^{m} v^{m}} \check{F}_{v^{m} \beta^{m}}^{m} \\
& +\sum_{v^{m}} U_{\alpha^{m}}^{m} v^{m} \tilde{g}_{v^{m} u^{m}}^{m} .
\end{aligned}
$$

For numerical reasons, the one-mode projector is implemented as

$$
\tilde{P}_{\alpha^{m} \beta^{m}}^{m}=\sum_{u^{m} v^{m}} U_{\alpha^{m} u^{m}}^{m}\left[\left(\tilde{\mathbf{S}}^{m}\right)^{-1}\right] u^{m} v^{m} U_{\beta^{m} v^{m}}^{m *},
$$

where $\tilde{S}_{u^{m} v^{m}}^{m}=\left[\tilde{a}_{u^{m}}^{m}, \tilde{a}_{v^{m}}^{m \dagger}\right]$ is the overlap matrix for mode $m$. This ensures that $P^{m}$ remains a projector if the time-dependent modals become non-orthonormal due to inexact numerical propagation of the EOMs.

\section{The mean-field operator for a sum-of-products Hamiltonian}

For a SOP Hamiltonian as defined in Eq. (8), the matrix elements of the mean-field operator can be evaluated as

$$
\begin{aligned}
\tilde{F}_{u^{m} r^{m}}^{m}= & \sum_{t \in\left\{t_{a c t}^{m}\right\}} c_{t}\left\langle\bar{\Psi}\left|\tilde{a}_{u^{m}}^{m \dagger}\left[\tilde{a}_{r^{m}}^{m}, \prod_{m \in \mathbf{m}_{t}} h^{m t}\right]\right| \bar{\Psi}\right\rangle \\
= & \sum_{t \in\left\{t_{a c t}^{m}\right\}} c_{t} \sum_{w^{m}} \sum_{\mathbf{v}}^{-m} C_{\mathbf{v}_{u^{m}}^{m}}^{*} \\
& \times\left(\sum_{\mathbf{w}}^{-m} C_{\mathbf{w}} \prod_{m^{\prime} \in \mathbf{m}_{t} \backslash m} \tilde{h}_{v^{m^{\prime}} w^{m^{\prime}}}^{m^{\prime}} \prod_{m^{\prime \prime} \notin \mathbf{m}_{t}} \delta_{v^{m^{\prime \prime}} w^{m^{\prime \prime}}}\right) \tilde{h}_{r^{m} w^{m}}^{m t} \\
= & \sum_{t \in\left\{t_{a c t}^{m}\right\}} c_{t} \sum_{w^{m}}\left(\sum_{\mathbf{v}}^{-m} C_{\mathbf{v}_{u^{m}}^{m}}^{*} T_{\mathbf{v}_{w^{m}}^{m}}^{m t}\right) \tilde{h}_{r^{m}}^{m t} w^{m} \\
= & \sum_{t \in\left\{t_{a c t}^{m}\right\}} c_{t} \sum_{w^{m}} \tilde{\mathcal{H}}_{u^{m}}^{m t} w^{m} \tilde{h}_{r^{m}}^{m t} w^{m},
\end{aligned}
$$

where $\left\{t_{a c t}^{m}\right\}$ only includes terms that operate on mode $m$. The $\mathbf{T}^{m t}$ tensor is constructed by performing $\operatorname{dim}\left(\mathbf{m}_{t}\right)-1$ one-index transformations on the $\mathbf{C}$ tensor. Contracting the tensors over all modes except $m$ results in the mean-field intermediates with elements $\tilde{\mathcal{H}}_{u^{m}}^{m t} v^{m}$. We note that the derivative of the configuration-space coefficients [Eq. (23)] can be obtained with almost no additional effort during the calculation of the mean-field intermediates.

The half-transformed matrix elements used in Eq. (34) are defined as

$$
\check{F}_{v^{m} \beta^{m}}^{m}=\sum_{r^{m}} \tilde{F}_{v^{m} r^{m}}^{m} U_{\beta^{m} r^{m}}^{m}=\sum_{t \in\left\{t_{a c t}^{m}\right\}} c_{t} \sum_{w^{m}} \tilde{\mathcal{H}}_{v^{m}}^{m t} w^{m} \check{h}_{\beta^{m}}^{m t} w^{m},
$$

where $\check{h}_{\beta^{m}}^{m t} w^{m}=\sum_{\alpha^{m}} h_{\beta^{m} \alpha^{m}}^{m t} U_{\alpha^{m}}^{m} w^{m}$ is obtained as a side product of transforming the Hamiltonian integrals to the time-dependent basis.

\section{THE MCTDH[ $n$ ] METHODS}

We now introduce the MCTDH $[n]$ hierarchy of methods for approximating the full MCTDH wave function in a systematic way. The general idea is to define a reference state $\left|\tilde{\Phi}_{\mathbf{i}}\right\rangle$ and include all configurations that are up to $n$-mode excited with respect to the reference (equivalent to the $\operatorname{VCI}[n]$ methods of Refs. 21 and 22).

We partition the active space for each mode into an occupied modal indexed by $i^{m}$ and $n^{m}-1$ virtual modals indexed $a^{m}, b^{m}$, and $c^{m}$. As discussed in Sec. III, the secondary modals defined as the orthogonal complement to the active space are indexed by $x^{m}$ and $y^{m}$. Furthermore, $u^{m}, v^{m}$, and $w^{m}$ denote active modals (occupied or 
virtual), while $r^{m}$ and $s^{m}$ are used to denote any modal (unspecified occupancy), and $\alpha^{m}$ and $\beta^{m}$ denote primitive-basis modals. See Fig. 1 for an illustration of the modal spaces.

The occupied modals are used for constructing the reference state,

$$
\left|\tilde{\Phi}_{\mathbf{i}}\right\rangle=\prod_{m} \tilde{a}_{i^{m}}^{m \dagger}|\mathrm{vac}\rangle
$$

from which we define excitations within the active space,

$$
\left|\tilde{\mathbf{a}}^{\mathbf{m}}\right\rangle=\tilde{\tau}_{\mathbf{a}^{\mathbf{m}}}^{\mathbf{m}}\left|\tilde{\Phi}_{\mathbf{i}}\right\rangle=\prod_{m \in \mathbf{m}} \tilde{a}_{a^{m}}^{m \dagger} \prod_{m^{\prime} \notin \mathbf{m}} \tilde{a}_{i^{m^{\prime}}}^{m^{\prime} \dagger}|\mathrm{vac}\rangle
$$

where $\mathbf{m} \equiv\left\{m_{0}, m_{1}, \ldots\right\}$ is a MC containing the excited modes.

The $\operatorname{MCTDH}[n]$ wave function is then written as

$$
|\bar{\Psi}\rangle=C_{\mathbf{i}}\left|\tilde{\Phi}_{\mathbf{i}}\right\rangle+\sum_{\mathbf{m} \in \operatorname{MCR}[I]} \sum_{\mathbf{a}^{\mathbf{m}}} C_{\mathbf{a}^{\mathbf{m}}}^{\mathbf{m}}\left|\tilde{\mathbf{a}}^{\mathbf{m}}\right\rangle,
$$

where $\mathrm{MCR}[I]$ is the mode-combination range (MCR) of the included configurations (excluding the empty set, i.e., the reference state that we have separated out explicitly). Including up to $n$-mode excitations in MCR $[I]$ defines the $\operatorname{MCTDH}[n]$ method, i.e., the sum is limited to MCs for which $\operatorname{dim}(\mathbf{m}) \leq n$. Thus, we do not restrict the configuration space based on a function of configuration indices (which can to some extent be thought of as quantum numbers) as done in some previous studies, ${ }^{17-19}$ i.e., the sum over $\mathbf{a}^{\mathbf{m}}$ is never truncated. The MCTDH $\left.n\right]$ wave function includes all possible configurations that are excited up to $n$ modes with respect to the reference configuration, and thus, the truncation is always performed in the sum over MCs. Note that MCR $[I]$ can in principle include any set of MCs, but here, we simply truncate the expansion based on one given excitation level. This criterion treats all modes equally, but it is theoretically fully possible to treat a subset of modes differently from the rest. Including selected higher-order excitations is an important subject for future investigation which will be pursued in forthcoming work.

\section{A. Derivation of the MCTDH[ $n$ ] EOMs}

From the MCTDH[n] wave-function Ansatz [Eq. (40)] we define the variation,

$$
|\delta \bar{\Psi}\rangle=\delta C_{\mathbf{i}}\left|\tilde{\Phi}_{\mathbf{i}}\right\rangle+C_{\mathbf{i}}\left|\delta \tilde{\Phi}_{\mathbf{i}}\right\rangle+\sum_{\mathbf{m} \in \operatorname{MCR}[I]} \sum_{\mathbf{a}^{\mathbf{m}}}\left(\delta C_{\mathbf{a}^{\mathbf{m}}}^{\mathbf{m}}\left|\tilde{\mathbf{a}}^{\mathbf{m}}\right\rangle+C_{\mathbf{a}^{\mathbf{m}}}^{\mathbf{m}}\left|\delta \tilde{\mathbf{a}}^{\mathbf{m}}\right\rangle\right)
$$

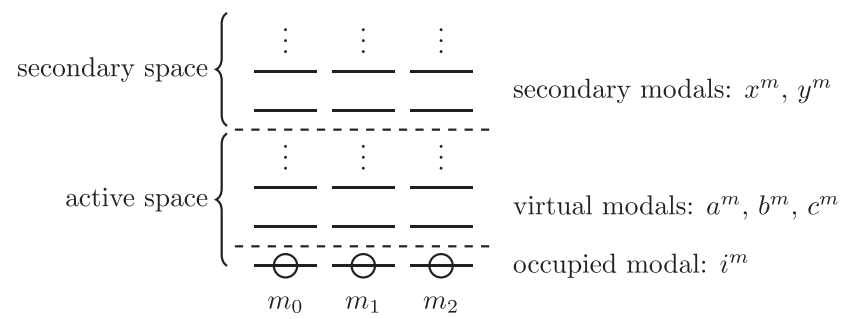

FIG. 1. Schematic representation of the reference configuration in MCTDH $[n]$ for a three-mode system with the modal spaces indicated. and the time derivative,

$$
|\dot{\Psi}\rangle=\dot{C}_{\mathbf{i}}\left|\tilde{\Phi}_{\mathbf{i}}\right\rangle+C_{\mathbf{i}}\left|\dot{\tilde{\Phi}}_{\mathbf{i}}\right\rangle+\sum_{\mathbf{m} \in \operatorname{MCR}[I]} \sum_{\mathbf{a}^{\mathbf{m}}}\left(\dot{C}_{\mathbf{a}^{\mathbf{m}}}^{\mathbf{m}}\left|\tilde{\mathbf{a}}^{\mathbf{m}}\right\rangle+C_{\mathbf{a}^{\mathbf{m}}}^{\mathbf{m}}\left|\dot{\tilde{\mathbf{a}}}^{\mathbf{m}}\right\rangle\right) .
$$

Using the Dirac-Frenkel TDVP, we obtain EOMs for the configuration-space coefficients,

$$
\begin{aligned}
i \dot{C}_{\mathbf{i}} & =\left\langle\tilde{\Phi}_{\mathbf{i}}|(H-g)| \bar{\Psi}\right\rangle, \\
i \dot{C}_{\mathbf{a}^{\mathbf{m}}}^{\mathbf{m}} & =\left\langle\tilde{\mathbf{a}}^{\mathbf{m}}|(H-g)| \bar{\Psi}\right\rangle,
\end{aligned}
$$

which are equivalent to (23). Varying the active-space modals yields,

$$
0=\left\langle\bar{\Psi}_{u^{m}}^{-m}\left|\delta \tilde{a}_{u^{m}}^{m}\left(H-i \frac{\partial}{\partial t}\right)\right| \bar{\Psi}\right\rangle .
$$

Applying the time derivative and inserting (43) and (44) results in

$$
\begin{aligned}
0= & \left\langle\bar{\Psi}_{u^{m}}^{-m}\left|\delta \tilde{a}_{u^{m}}^{m}(H-g)\right| \bar{\Psi}\right\rangle-i\left\langle\bar{\Psi}_{u^{m}}^{-m}\right| \delta \tilde{a}_{u^{m}}^{m} \\
& \times\left(\dot{C}_{\mathbf{i}}\left|\tilde{\Phi}_{\mathbf{i}}\right\rangle+\sum_{\mathbf{m} \in \mathrm{MCR}[I]} \sum_{\mathbf{a}^{\mathbf{m}}} \dot{C}_{\mathbf{a}^{\mathbf{m}}}^{\mathbf{m}}\left|\tilde{\mathbf{a}}^{\mathbf{m}}\right\rangle\right) \\
= & \left\langle\bar{\Psi}_{u^{m}}^{-m}\left|\delta \tilde{a}_{u^{m}}^{m}\left(I-\tilde{P}_{I}\right)(H-g)\right| \bar{\Psi}\right\rangle \\
= & \left\langle\bar{\Psi}_{u^{m}}^{-m}\left|\delta \tilde{a}_{u^{m}}^{m}(I-P)(H-g)\right| \bar{\Psi}\right\rangle+\left\langle\bar{\Psi}_{u^{m}}^{-m}\left|\delta \tilde{a}_{u^{m}}^{m} \tilde{P}_{X}(H-g)\right| \bar{\Psi}\right\rangle,
\end{aligned}
$$

where we have introduced the projection operators,

$$
\begin{aligned}
P= & \tilde{P}_{I}+\tilde{P}_{X}=\underbrace{\left|\tilde{\Phi}_{\mathbf{i}}\right\rangle\left\langle\tilde{\Phi}_{\mathbf{i}}\left|+\sum_{\mathbf{m} \in \operatorname{MCR}[I] \mathbf{a}^{\mathbf{m}}}\right| \tilde{\mathbf{a}}^{\mathbf{m}}\right\rangle\left\langle\tilde{\mathbf{a}}^{\mathbf{m}}\right|}_{\tilde{P}_{I}} \\
& +\underbrace{\sum_{\mathbf{m}^{\prime} \in \operatorname{MCR}[X] \mathbf{b}^{\mathbf{m}^{\prime}}}\left|\tilde{\mathbf{b}}^{\mathbf{m}^{\prime}}\right\rangle\left\langle\tilde{\mathbf{b}}^{\mathbf{m}^{\prime}}\right|,}_{\tilde{P}_{X}},
\end{aligned}
$$

and defined MCR $[X]$ as the set of excluded mode combinations, i.e., those not accounted for explicitly in the wave function. The first term on the RHS of Eq. (46) yields the standard MCTDH modal EOMs [Eq. (30)]. Note that the density matrices and mean fields are of course calculated differently in $\operatorname{MCTDH}[n]$ (see Sec. IV C). This term can only be non-zero if the variation is orthogonal to the active space, i.e., $\delta \tilde{a}_{u^{m}}^{m}=\sum_{x^{m}} k_{x^{m}} \tilde{a}_{x^{m}}^{m}$. The second term can only be non-zero if the variation is contained in the active space, i.e., $\delta \tilde{a}_{u^{m}}^{m}=k_{i^{m}} \tilde{a}_{i^{m}}^{m}+\sum_{a^{m}} k_{a^{m}} \tilde{a}_{a^{m}}^{m}$, and thus, both terms need to be zero individually. The second term defines a linear set of equations for determining the variationally optimal $g$ operator as will be derived and discussed in Sec. IV B.

\section{B. Variational optimization of the constraint operators}

Setting the second term of (46) equal to zero results in

$$
0=\sum_{\mathbf{m}^{\prime} \in \mathrm{MCR}[X]} \sum_{\mathbf{b}^{\mathbf{m}^{\prime}}}\langle\underbrace{\left\langle\bar{\Psi}\left|\tilde{a}_{u^{m}}^{m \dagger} \delta \tilde{a}_{u^{m}}^{m}\right| \tilde{\mathbf{b}}^{\mathbf{m}^{\prime}}\right\rangle}_{A} \underbrace{\left\langle\left\langle\tilde{\mathbf{b}}^{\mathbf{m}^{\prime}}|H| \bar{\Psi}\right\rangle-\left\langle\tilde{\mathbf{b}}^{\mathbf{m}^{\prime}}|g| \bar{\Psi}\right\rangle\right)}_{B} .
$$

Factor $A$ can be evaluated by using the fact that the operator $\tilde{E}_{i^{m} i^{m}}^{m}+\tilde{E}_{b^{m} b^{m}}^{m}$ acts as the identity when applied to the $\left|\tilde{\mathbf{b}}^{\mathbf{m}^{\prime}}\right\rangle$ state, i.e., 
mode $m$ is either occupied ( $m \notin \mathbf{m}^{\prime}$ ) or excited to modal $b^{m}$

$$
\begin{aligned}
A: & \left\langle\bar{\Psi}\left|\tilde{a}_{u^{m}}^{m \dagger} \delta \tilde{a}_{u^{m}}^{m}\left(\tilde{E}_{i^{m} i^{m}}^{m}+\tilde{E}_{b^{m} b^{m}}^{m}\right)\right| \tilde{\mathbf{b}}^{\mathbf{m}^{\prime}}\right\rangle \\
= & {\left[\delta \tilde{a}_{u^{m}}^{m}, \tilde{a}_{i^{m}}^{m \dagger}\right]\left\langle\bar{\Psi}\left|\tilde{E}_{u^{m} i^{m}}^{m}\right| \tilde{\mathbf{b}}^{\mathbf{m}^{\prime}}\right\rangle+\left[\delta \tilde{a}_{u^{m}}^{m}, \tilde{a}_{b^{m}}^{m \dagger}\right]\left\langle\bar{\Psi}\left|\tilde{E}_{u^{m} b^{m}}^{m}\right| \tilde{\mathbf{b}}^{\mathbf{m}^{\prime}}\right\rangle . }
\end{aligned}
$$

We note that $u^{m}$ must be virtual in the first term and occupied in the second in order to get a non-zero contribution. The $B$ term is evaluated by noting that only the parts of the $g$ operator that shift occupation between occupied and virtual modals give non-zero contributions,

$$
B: \quad \mathcal{K}_{\mathbf{b}^{\mathbf{m}^{\prime}}}^{\mathbf{m}^{\prime}}-\sum_{m^{\prime}} \sum_{a^{m^{\prime}}}\left(\left\langle\tilde{\mathbf{b}}^{\mathbf{m}^{\prime}}\left|\tilde{E}_{i^{m^{\prime}} a^{m^{\prime}}}^{m^{\prime}}\right| \bar{\Psi}\right\rangle \tilde{g}_{i^{m^{\prime}}}^{m^{\prime} a^{m^{\prime}}}+\left\langle\tilde{\mathbf{b}}^{\mathbf{m}^{\prime}}\left|\tilde{E}_{a^{m^{\prime}} i^{m^{\prime}}}^{m^{\prime}}\right| \bar{\Psi}\right\rangle \tilde{g}_{a^{m^{\prime}} i^{m^{\prime}}}^{m^{\prime}}\right),
$$

with $\mathcal{K}_{\mathbf{b}^{\mathbf{m}^{\prime}}}^{\mathbf{m}^{\prime}} \equiv\left\langle\tilde{\mathbf{b}}^{\mathbf{m}^{\prime}}|H| \bar{\Psi}\right\rangle$. If MCR $[I]$ is closed under one-mode deexcitation (i.e., one cannot de-excite from an included to an excluded configuration), the first term in (49) and the middle term in (50) vanish. Inserting this into (48) results in

$$
\begin{aligned}
0= & \sum_{\mathbf{m}^{\prime} \in \operatorname{MCR}\left[X_{1}\right]} \sum_{\mid m \in \mathbf{m}^{\prime}}\left[\delta \tilde{a}_{i^{m}}^{m}, \tilde{a}_{b^{m}}^{m \dagger}\right] \sum_{\mathbf{b}^{\mathbf{m}^{\prime}}}^{-m}\left\langle\bar{\Psi}\left|\tilde{E}_{i^{m} b^{m}}^{m}\right| \tilde{\mathbf{b}}^{\mathbf{m}^{\prime}}\right\rangle \\
& \times\left(\mathcal{K}_{\mathbf{b}^{\mathbf{m}^{\prime}}}^{\mathbf{m}^{\prime}}-\sum_{m^{\prime} \in \mathbf{m}^{\prime}}\left\langle\tilde{\mathbf{b}}^{\mathbf{m}^{\prime}}\left|\tilde{E}_{b^{m^{\prime}} i^{m^{\prime}}}^{m^{\prime}}\right| \bar{\Psi}\right\rangle \tilde{g}_{b^{m^{\prime}} i^{m^{\prime}}}^{m^{\prime}}\right),
\end{aligned}
$$

where we have defined MCR $\left[X_{1}\right]$ as the set of excluded MCs that are one-mode excited with respect to an included MC. Assuming independent variations (i.e., setting $\delta \tilde{a}_{i^{m}}^{m}=\sum_{u^{m}} k_{u^{m}} \tilde{a}_{u^{m}}^{m}$, where $k_{u^{m}}$ can be chosen arbitrarily), this becomes

$$
\begin{aligned}
0= & \sum_{\mathbf{m}^{\prime} \in \operatorname{MCR}\left[X_{1}\right]} \sum_{m \in \mathbf{m}^{\prime} \mathbf{b}^{\mathbf{m}^{\prime}}} \sum^{-m}\left\langle\bar{\Psi}\left|\tilde{E}_{i^{m} b^{m}}^{m}\right| \tilde{\mathbf{b}}^{\mathbf{m}^{\prime}}\right\rangle \\
& \times\left(\mathcal{K}_{\mathbf{b}^{\mathbf{m}^{\prime}}}^{\mathbf{m}^{\prime}}-\sum_{m^{\prime} \in \mathbf{m}^{\prime}}\left\langle\tilde{\mathbf{b}}^{\mathbf{m}^{\prime}}\left|\tilde{E}_{b^{m^{\prime}} i^{m^{\prime}}}^{m^{\prime}}\right| \bar{\Psi}\right\rangle \tilde{g}_{b^{m^{\prime}} i^{m^{\prime}}}^{m^{\prime}}\right) .
\end{aligned}
$$

Using $\left\langle\tilde{\mathbf{b}}^{\mathbf{m}^{\prime}}\left|\tilde{E}_{b^{m} m^{m}}^{m}\right| \bar{\Psi}\right\rangle=C_{\mathbf{b}^{\mathbf{m}^{\prime}} \backslash m}^{\mathbf{m}^{\prime} \backslash m}$, this can be re-written as a set of linear equations for determining the virtual-occupied blocks of the constraint operators,

$$
\sum_{m^{\prime}} \sum_{b^{m^{\prime}}} \mathcal{A}_{a^{m} b^{m^{\prime}}}^{m m^{\prime}} \tilde{g}_{b^{m^{\prime}} i^{m^{\prime}}}^{m^{\prime}}=\mathcal{B}_{a^{m}}^{m}
$$

where we have defined

$$
\begin{gathered}
\mathcal{A}_{b^{m} b^{m^{\prime}}}^{m m^{\prime}} \equiv \sum_{\mathbf{m}^{\prime} \in \mathrm{MCR}\left[X_{1}\right] \mid m, m^{\prime} \in \mathbf{m}^{\prime}} \sum_{\mathbf{b}^{\mathbf{m}^{\prime}}}^{-m, m^{\prime}} C_{\mathbf{b}^{\mathbf{m}^{\prime} \backslash m}}^{\mathbf{m}^{\prime} \backslash m^{*}} C_{\mathbf{b}^{\mathbf{m}^{\prime} \backslash m^{\prime}}}^{\mathbf{m}^{\prime}, m^{\prime}} \\
\mathcal{B}_{b^{m}}^{m} \equiv \sum_{\mathbf{m}^{\prime} \in \operatorname{MCR}\left[X_{1}\right] \mid m \in \mathbf{m}^{\prime}} \sum_{\mathbf{b}^{\mathbf{m}^{\prime}}}^{-m} C_{\mathbf{b}^{\mathbf{m}^{\prime} \backslash m}}^{\mathbf{m}^{\prime} \backslash m^{*}} \mathcal{K}_{\mathbf{b}^{\mathbf{m}^{\prime}}}^{\mathbf{m}^{\prime}}
\end{gathered}
$$

Equation (53) is a set of linear equations of dimension $N_{v i r}^{\text {tot }}$ $\times N_{v i r}^{\text {tot }}$ with $N_{v i r}^{\text {tot }} \equiv \sum_{m}\left(n^{m}-1\right)$ for determining the virtual-occupied blocks of the $\tilde{\mathbf{g}}^{m}$ matrices. All other elements are redundant and can in principle be chosen arbitrarily (i.e., set to zero), but we require the constraint operators to be Hermitian in order to conserve orthonormality of the time-dependent modals and, thus, set the occupied-virtual elements accordingly. Note that this can only be done if the MCR $[I]$ is closed under one-mode de-excitation. Otherwise, the term in (50) depending on $\tilde{g}_{i^{m} a^{m}}^{m}$ could not be eliminated and Hermiticity would have to be enforced making the Lagrange and McLachlan TDVPs inequivalent (see Ref. 33).

Solving (53), optimizes the occupied modal in each mode such that the reference for the truncated CI expansion becomes optimal for representing the wave function. Note that the equations are also defined in the limit $n^{m}=N^{m} \forall m$, i.e., the off-diagonal blocks of the $\tilde{\mathbf{g}}^{m}$ matrices are also non-redundant when the active space includes all modals for each mode. Using (53), for obtaining $\tilde{g}_{b^{m} i^{m}}^{m}$, elements require some consideration, however. First of all, explicit calculation of the $\mathcal{K}_{\mathbf{b}^{\mathrm{m}}}^{\mathbf{m}}$ intermediate for constructing the $\mathcal{B}_{a^{m}}^{m}$ vector is computationally expensive and will become a bottleneck. In our implementation, this is solved by avoiding the construction of $\mathcal{K}_{\mathbf{b}^{\mathrm{m}}}^{\mathbf{m}}$ and combining the Hamiltonian transformation and the contraction with the $C$ coefficients (see Sec. V). Second, the $\mathcal{A}$ matrix is not guaranteed to be non-singular and the problem may become ill-posed and require regularization as discussed in Sec. V. Furthermore, for systems with many degrees of freedom, solving Eq. (53) may become costly. These potential issues lead us to examine another choice of constraint which keeps the density matrices block diagonal in all modes (see Sec. IV C).

As a final perspective, Eq. (53) can also be derived as a LLS minimization of the time derivative of the $(n+1)$-mode excitations for $\mathrm{MCTDH}[n]$. Considering the EOM for the coefficients of $\mathbf{m}^{\prime} \in \operatorname{MCR}\left[X_{1}\right]$,

$$
i \dot{C}_{\mathbf{b}^{\mathbf{m}^{\prime}}}^{\mathbf{m}^{\prime}}=\left\langle\tilde{\mathbf{b}}^{\mathbf{m}^{\prime}}|(H-g)| \bar{\Psi}\right\rangle
$$

Setting this expression equal to zero and assuming that MCR $[I]$ is closed under one-mode de-excitation results in an overdetermined linear system of equations,

$$
\sum_{m} \sum_{a^{m}}\left\langle\tilde{\mathbf{b}}^{\mathbf{m}^{\prime}}\left|\tilde{E}_{a^{m} i^{m}}^{m}\right| \bar{\Psi}\right\rangle \tilde{g}_{a^{m} i^{m}}^{m}=\mathcal{K}_{\mathbf{b}^{\mathbf{m}^{\prime}}}^{\mathbf{m}^{\prime}}
$$

Solving this using normal equations yields Eq. (53). Thus, the variational constraint operators are seen to minimize the time evolution of the excluded configurations that are one-mode excited with respect to an included configuration.

\section{Density matrices and natural-modal constraints}

The blocks of the MCTDH $[n]$ density matrices are evaluated as

$$
\begin{aligned}
& \tilde{D}_{i^{m} i^{m}}^{m}=\left|C_{\mathbf{i}}\right|^{2}+\sum_{\mathbf{m}^{\prime} \in \operatorname{MCR}[I] \mid m \notin \mathbf{m}^{\prime} \mathbf{a}^{\mathbf{m}^{\prime}}}\left|C_{\mathbf{a}^{\mathbf{m}^{\prime}}}^{\mathbf{m}^{\prime}}\right|^{2}, \\
& \tilde{D}_{a^{m} i^{m}}^{m}=\sum_{\mathbf{m}^{\prime} \in \operatorname{MCR}[I] \mid} \sum_{m \in \mathbf{m}^{\prime}} \sum_{\mathbf{b}^{\mathbf{m}^{\prime}}}^{-m} C_{\mathbf{b}_{m, a^{m}}^{\mathbf{m}^{\prime}}}^{\mathbf{m}^{\prime}}{ }^{*} C_{\mathbf{b}^{\mathbf{m}^{\prime}} \backslash m}^{\mathbf{m}^{\prime} \backslash m}, \\
& \tilde{D}_{a^{m} b^{m}}^{m}=\sum_{\mathbf{m}^{\prime} \in \operatorname{MCR}[I] \mid m \in \mathbf{m}^{\prime}} \sum_{\mathbf{c}^{\mathbf{m}^{\prime}}}^{-m} C_{\mathbf{c}_{m, a^{m}}^{\mathbf{m}^{\prime}}}^{\mathbf{m}^{\prime}}{ }^{*} C_{\mathbf{c}_{m, b^{m}}^{\mathbf{m}^{\prime}}}^{\mathbf{m}^{\prime}},
\end{aligned}
$$

where $C_{\mathbf{b}_{m, a^{m}}^{\mathbf{m}^{\prime}}}^{\mathbf{m}^{\prime}}$ has indices $b^{m^{\prime}} \forall m^{\prime} \in \mathbf{m}^{\prime} m$ and index $a^{m}$ for mode $m$. Note that for $\mathbf{m}^{\prime}=\{m\}, C_{\mathbf{b}^{\mathbf{m}^{\prime}} \backslash m}^{\mathbf{m}^{\prime} \backslash m}=C$. 
It is now relevant to consider a choice of constraint operators that keeps the one-mode density matrices diagonal (or block diagonal) during the propagation. For MCTDH, the choice of natural modals does not change the wave function, ${ }^{3,41}$ but for approximate $\operatorname{MCTDH}[n]$, it seems reasonable to choose natural modals for defining the reference state. ${ }^{17,18,42}$ As shown in Sec. IV B, only the virtual-occupied blocks of the $g^{m}$ operators are non-redundant and, thus, able to change the wave function, i.e., rotations among the virtual modals do not affect the accuracy of the results. Thus, we now derive equations for determining the $g^{m}$ operators such that the density matrices become block diagonal, i.e., $\tilde{D}_{a^{m} i^{m}}^{m}=0$ at all times.

If the propagation starts from a set of natural occupied modals [or perhaps iterative natural modals (ItNaMos) ${ }^{43}$ ], we require,

$$
\begin{aligned}
0=\dot{\tilde{D}}_{a^{m} i^{m}}^{m}= & \sum_{\mathbf{m}^{\prime} \in \operatorname{MCR}[I]} \sum_{\mid m \in \mathbf{m}^{\prime} \mathbf{b}^{\mathbf{m}^{\prime}}}^{-m} \\
& \times\left(C_{\mathbf{b}_{m, a m}^{\mathbf{m}^{\prime}}}^{\mathbf{m}^{\prime}}{ }^{*} \dot{C}_{\mathbf{b}^{\mathbf{m}^{\prime} \backslash m}}^{\mathbf{m}^{\prime} \backslash m}+\left(C_{\mathbf{b}^{\mathbf{m}^{\prime} \backslash m}}^{\mathbf{m}^{\prime} \backslash m^{*}} \dot{C}_{\mathbf{b}_{m, a m}^{\mathbf{m}^{\prime}}}^{\mathbf{m}^{\prime}}\right)^{*}\right) \\
= & -i \sum_{\mathbf{m}^{\prime} \in \mathrm{MCR}[I] \mid} \sum_{m \in \mathbf{m}^{\prime} \mathbf{b}^{\mathbf{m}^{\prime}}}{ }^{-m}\left(C_{\mathbf{b}_{m, a^{m}}^{\mathbf{m}^{\prime}}}^{\mathbf{m}^{\prime}}{ }^{*}\left\langle\tilde{\mathbf{b}}^{\mathbf{m}^{\prime} \backslash m}|(H-g)| \bar{\Psi}\right\rangle\right. \\
& \left.-\left(C_{\mathbf{b}^{\mathbf{m}^{\prime} \backslash m}}^{\mathbf{m}^{\prime} \backslash m^{*}}\left\langle\tilde{\mathbf{b}}_{m, a^{m}}^{\mathbf{m}^{\prime}}|(H-g)| \bar{\Psi}\right\rangle\right)^{*}\right) \\
= & i\left\langle\bar{\Psi}\left|\left[H-g, \tilde{E}_{a^{m} i^{m}}^{m}\right]\right| \bar{\Psi}\right\rangle .
\end{aligned}
$$

This leads to

$$
\left\langle\bar{\Psi}\left|\left[H, \tilde{E}_{a^{m} i^{m}}^{m}\right]\right| \bar{\Psi}\right\rangle=\left\langle\bar{\Psi}\left|\left[g^{m}, \tilde{E}_{a^{m} i^{m}}^{m}\right]\right| \bar{\Psi}\right\rangle,
$$

where the LHS reduces to

$$
\begin{aligned}
\left\langle\bar{\Psi}\left|\left[H, \tilde{E}_{a^{m} i^{m}}^{m}\right]\right| \bar{\Psi}\right\rangle= & \sum_{t \in\left\{t_{a c t}^{m}\right\}} c_{t} \sum_{u^{m}}\left(\tilde{h}_{u^{m} a^{m}}^{m t}\left\langle\bar{\Psi}\left|\tilde{E}_{u^{m} i^{m}}^{m} \prod_{m^{\prime} \in \mathbf{m}_{t} \backslash m} h^{m^{\prime} t}\right| \bar{\Psi}\right\rangle\right. \\
& \left.-\tilde{h}_{i^{m} u^{m}}^{m t}\left\langle\bar{\Psi}\left|\tilde{E}_{a^{m} u^{m}}^{m} \prod_{m^{\prime} \in \mathbf{m}_{t} \backslash m} h^{m^{\prime} t}\right| \bar{\Psi}\right\rangle\right) \\
= & \tilde{F}_{i^{m} a^{m}}^{m *}-\tilde{F}_{a^{m} i^{m}}^{m},
\end{aligned}
$$

and the RHS becomes

$$
\begin{aligned}
& \sum_{u^{m} v^{m}} \tilde{g}_{u^{m} v^{m}}^{m}\left\langle\bar{\Psi}\left|\left[\tilde{E}_{u^{m} v^{m}}^{m}, \tilde{E}_{a^{m} i^{m}}^{m}\right]\right| \bar{\Psi}\right\rangle \\
& =\sum_{b^{m}}\left(\delta_{a^{m} b^{m}} \tilde{D}_{i^{m} i^{m}}^{m}-\tilde{D}_{a^{m} b^{m}}^{m}\right) \tilde{g}_{i^{m} b^{m}}^{m},
\end{aligned}
$$

where we have used the assumption that $\tilde{D}_{a^{m} i^{m}}^{m}=0$. Note that this assumption may become invalid due to inexact propagation of the EOMs.

Thus, the constraint operators for propagation in natural occupied modals can be obtained by solving the linear equations,

$$
\sum_{b^{m}}\left(\delta_{a^{m} b^{m}} \tilde{D}_{i^{m} i^{m}}^{m}-\tilde{D}_{a^{m} b^{m}}^{m}\right) \tilde{g}_{i^{m} b^{m}}^{m}=\tilde{F}_{i^{m} a^{m}}^{m *}-\tilde{F}_{a^{m} i^{m}}^{m},
$$

for all $m$. Propagating in the full set of natural modals and not just occupied natural modals is also possible by choosing the $\tilde{g}_{a^{m} b^{m}}^{m}$ ele-

\begin{tabular}{|c|c|}
\hline MCTDH[n,g0] & $g=0$ \\
\hline Benefits & $\begin{array}{l}\text { - Simple } \\
\text { - The choice of } g \text { is never singular } \\
\text { - Minimizes the time evolution of the modals, which enables the use of a } \\
\text { constant mean-field (CMF) integrator }\end{array}$ \\
\hline Drawbacks & $\begin{array}{l}\text { - Not variational } \\
\text { - The occupied modals are not optimized with respect to rotations within the } \\
\text { active space }\end{array}$ \\
\hline $\begin{array}{l}\text { MCTDH[n,D] } \\
\text { Benefits } \\
\text { Drawbacks }\end{array}$ & $\begin{array}{l}\text { Obtain } g \text { by solving Eq. }(65) \\
\text { - Makes the reference state a product of natural modals with high occupation } \\
\text { - May become singular and require regularization }\end{array}$ \\
\hline $\begin{array}{l}\text { MCTDH[n,V] } \\
\text { Benefits } \\
\text { Drawbacks }\end{array}$ & $\begin{array}{l}\text { Obtain } g \text { by solving Eq. (53) } \\
\text { - Fully variational solution to the time-dependent Schrödinger equation } \\
\text { - Computationally expensive } \\
\text { - May become singular and require regularization }\end{array}$ \\
\hline
\end{tabular}
ments such that the $\tilde{D}_{a^{m} b^{m}}^{m}$ matrix is diagonal (see Refs. 3 and 41). The linear equations in Eq. (65) become singular if one of the virtual modals gets the same natural occupation number as the occupied modal, i.e., if one of the eigenvalues of the virtual-virtual block becomes equal to $\tilde{D}_{i^{m} i^{m}}^{m}$. Thus, Eq. (65) needs to be regularized in order to ensure a stable propagation. ${ }^{3}$ Note, however, that the block-diagonal constraint does not give rise to singular constraint operators if two virtual modals get the same natural occupation as is the case when propagating in all natural modals.

TABLE I. Overview of the different choices of $g$ and their benefits and drawbacks. 
The question now is whether this choice of $g^{m}$ is better than the variationally optimized one derived in Sec. IV B. The TDVP is no longer satisfied, but the occupation in the occupied modals is kept as large as possible during the propagation. Also, the naturalmodal constraint is simpler and computationally cheaper than the fully variational constraint. See Table I for a summary of the various choices of $g$.

\section{Separability}

The MCTDH $[n]$ wave function is based on a linear Ansatz like VCI, and it is therefore relevant to discuss the features of the theory with respect to separability of non-interacting subsystems. It is wellknown in the time-independent theory that the exact wave function for an additively separable Hamiltonian, $H=H_{A}+H_{B}$, is multiplicatively separable, $|\alpha\rangle=\left|\alpha_{A} \alpha_{B}\right\rangle$, and the energy is additively separable, $E=E_{A}+E_{B} \cdot{ }^{37}$ Likewise, in the time-dependent case, the EOMs for the different subsystems should decouple.

Consider the MCTDH $[n]$ wave functions for the subsystems $A$ and $B$,

$$
\left|\bar{\Psi}_{A}\right\rangle=\left(C_{\mathbf{i} A}+\hat{C}_{A}\right) \tilde{W}_{A}|\mathrm{vac}\rangle \quad\left|\bar{\Psi}_{B}\right\rangle=\left(C_{\mathbf{i} B}+\hat{C}_{B}\right) \tilde{W}_{B}|\mathrm{vac}\rangle,
$$

where $\tilde{W}_{A}|\mathrm{vac}\rangle=\left|\tilde{\Phi}_{\mathbf{i}}^{A}\right\rangle$ is the reference configuration for subsystem $A$ and $\hat{C}_{A}=\sum_{\mathbf{a}_{A}} C_{\mathbf{a}_{A}}^{A} \tilde{\tau}_{\mathbf{a}_{A}}^{A}$ creates the excitations. The total wave function is then

$$
\begin{aligned}
|\bar{\Psi}\rangle & =\left(C_{\mathbf{i} A}+\hat{C}_{A}\right) \tilde{W}_{A}\left(C_{\mathbf{i} B}+\hat{C}_{B}\right) \tilde{W}_{B}|\mathrm{vac}\rangle \\
& =\left(C_{\mathbf{i} A} C_{\mathbf{i} B}+C_{\mathbf{i} A} \hat{C}_{B}+C_{\mathbf{i} B} \hat{C}_{A}+\hat{C}_{A} \hat{C}_{B}\right)\left|\tilde{\Phi}_{\mathbf{i}}\right\rangle .
\end{aligned}
$$

If the wave functions of the subsystems are truncated at $n$-mode excitations, the total wave function includes up to $2 n$-mode excitations and is, thus, not representable within the MCTDH $[n]$ model. This problem becomes more pronounced as the number of subsystems increases, and thus, it is expected that higher excitation levels are required in order to obtain the same accuracy for larger systems. However, if the time evolution of the modals is chosen such that the weights of the reference configurations are kept as large as possible during the propagation of the EOMs, the importance of the $\hat{C}_{A} \hat{C}_{B}$ term is kept small which results in smaller errors.

We note that for the time-independent theory the VCC model behaves correctly with respect to separability. This has recently been extended to the time-dependent context in Ref. 26 where a detailed discussion of the separability properties of TDVCC is given.

\section{IMPLEMENTATION}

The $\operatorname{MCTDH}[n]$ methods together with the full MCTDH method have been implemented in the MidasCpp program, ${ }^{45}$ which also features efficient implementations of $\mathrm{TDH}^{4}$ and TDVCC, ${ }^{26}$ an array of methods for time-independent vibrational-structure calculations, ${ }^{23,25,35}$ tools for automatic PES generation, ${ }^{46-48}$ coordinate optimization, ${ }^{49,50}$ etc. The MCTDH module has been implemented to interact with the time-independent wave-function modules of MidasCpp such that it can obtain initial wave functions and modal bases from prior vibrational self-consistent field (VSCF), ${ }^{35,51,52} \mathrm{VCI}$, and MCTDH calculations.
The MCTDH EOMs are propagated in time using the generalpurpose routines introduced in Ref. 4 . For all calculations in this work, we use the Dormand-Prince 8(5,3) explicit Runge-Kutta method $^{53}$ with adaptive step-size control (medium-tight tolerance) as well as dense output for obtaining equidistant values of the autocorrelation function.

In order to entirely avoid the risk of the matrices used for optimizing the $\tilde{g}_{a^{m} i^{m}}^{m}$ elements becoming near-singular, a smooth regularization scheme like the one used for inverting density matrices $^{3}$ is always used in solving Eqs. (53) and (65). The matrices are diagonalized and the eigenvalues are regularized as

$$
\lambda_{\text {reg }}=\lambda+\epsilon \exp (-\lambda / \epsilon)
$$

before constructing the inverse. The choice of $\epsilon$ is not very critical, and in this work, we use $\epsilon=10^{-12}$ for $\operatorname{MCTDH}[n, \mathrm{~V}]$ and $\epsilon=10^{-6}$ for $\operatorname{MCTDH}[n, \mathrm{D}]$.

The mean-field intermediates and the derivative of the configuration-space coefficients are in both $\operatorname{MCTDH}[n]$ and the full MCTDH implementation calculated by looping over terms in the Hamiltonian. The wave-function coefficients are then transformed with the one-mode operators one by one (using a specialized routine for $\mathrm{MCTDH}[n]$ ). After each transformation, the following one-mode operator is skipped and the remaining transformations are performed in order to construct the $\mathbf{T}^{\text {mt }}$ coefficients used for calculating $\mathbf{H}^{m t}$ [see Eq. (36)]. The loop over operator terms has been parallelized using OpenMP. The one-mode transformation in $\operatorname{MCTDH}[n]$ is implemented by partitioning of each one-mode operator into four types of one-index contractions, passive, up, down, and forward, ${ }^{24,54}$

$$
\begin{aligned}
h^{m t} & =\tilde{h}_{i^{m} i^{m}}^{m t} \tilde{E}_{i^{m} i^{m}}^{m}+\sum_{a^{m}} \tilde{h}_{a^{m} i^{m}}^{m t} \tilde{E}_{a^{m} i^{m}}^{m}+\sum_{a^{m}} \tilde{h}_{i^{m} a^{m}}^{m t} \tilde{E}_{i^{m} a^{m}}^{m}+\sum_{a^{m} b^{m}} \tilde{h}_{a^{m} b^{m}}^{m t} \tilde{E}_{a^{m} b^{m}}^{m} \\
& =h_{p}^{m t}+h_{u}^{m t}+h_{d}^{m t}+h_{f}^{m t} .
\end{aligned}
$$

Ideally, down contractions should be applied first followed by forward and up in order to obtain the lowest computational cost. However, in order to calculate mean fields, we perform all contractions belonging to one operator before proceeding to the next instead of swapping them. Integrating the mean-field (and $\mathcal{B}$ vector) calculation in the general framework described in Ref. 24 with automatic identification and exploitation of intermediates is a topic for future investigation.

The $\mathcal{B}_{a^{m}}^{m}$ elements are calculated by considering all combinations of contraction types for each term in the Hamiltonian that are able to create a one-mode excited configuration from an included configuration. Instead of applying the up contractions to the ket coefficients (which would result in a tensor of higher dimensionality), they are evaluated as down contractions on the $C_{\mathbf{b}^{\mathbf{m}^{\prime}} \backslash m}^{\mathbf{m}^{\prime} m^{*}}$ coefficients [see Eq. (55)]. In this way, no tensors of higher order than $n$ are constructed for $\operatorname{MCTDH}[n, \mathrm{~V}]$.

\section{RESULTS}

\section{A. Computational details}

In the following, the numerical results for the $\operatorname{MCTDH}[n]$ methods with different choices of constraint operators are 
presented. Calculations are performed on formyl fluoride and a series of high-dimensional Henon-Heiles potentials,

$H_{\text {Henon-Heiles }}=-\frac{1}{2} \sum_{m=1}^{M} \frac{\partial^{2}}{\partial q_{m}^{2}}+\frac{1}{2} \sum_{m=1}^{M} q_{m}^{2}+\lambda \sum_{m=1}^{M-1}\left(q_{m}^{2} q_{m+1}-\frac{1}{3} q_{m+1}^{3}\right)$,

with $\lambda=0.111803$. The formyl fluoride PES has been calculated using the adaptive density-guided approach (ADGA), including up to three-mode couplings with relative and absolute thresholds, $\epsilon_{\text {rel }}=10^{-2}$ and $\epsilon_{\text {abs }}=10^{-6}$. The electronicstructure points are obtained from the TURBOMOLE program. ${ }^{5}$

The one and two-mode parts of the PES have been calculated using coupled cluster with singles, doubles, and scaled perturbative triples $\left[\operatorname{CCSD}\left(\mathrm{F} 12^{*}\right)\left(\mathrm{T}^{*}\right)\right]^{56}$ and the correlation consistent double- $\zeta$ basis set optimized for explicitly correlated methods (ccpVDZ-F12). ${ }^{57}$ The three-mode part has been calculated using the resolution-of-the-identity 2nd-order Møller-Plesset perturbation theory (RI-MP2) ${ }^{58,59}$ and the correlation consistent triple- $\zeta$ basis set (cc-pVTZ). ${ }^{60}$ The frozen-core approximation has been used in the calculation of the correlation energy. The electronic-structure points have been fitted using up to 12th-order polynomials for each mode (the coupling terms have a maximum combined polynomial order of 12). The final PES contains 2667 terms and is provided as the supplementary material.

All $\operatorname{MCTDH}[n]$ calculations are performed using a B-spline basis set, ${ }^{61}$ which is transformed into a set of orthonormal VSCF modals before starting the propagation. In all calculations presented here, a VSCF wave function is used as initial wave packet.

In order to quantify differences between MCTDH wave functions, we use the squared difference norm,

$$
\left\|\Psi_{1}-\Psi_{2}\right\|^{2}=\left\|\Psi_{1}\right\|^{2}+\left\|\Psi_{2}\right\|^{2}-2 \operatorname{Re}\left\langle\Psi_{1} \mid \Psi_{2}\right\rangle,
$$

and the Hilbert-space angle,

$$
\vartheta=\arccos \left(\frac{\left|\left\langle\Psi_{1} \mid \Psi_{2}\right\rangle\right|}{\left\|\Psi_{1}\right\|\left\|\Psi_{2}\right\|}\right) .
$$

The latter is only sensitive to the shape of the wave function and not to phase and norm errors.

Spectra are obtained by performing fast Fourier transform (FFT) on the autocorrelation function,

$$
S(t)=\langle\bar{\Psi}(0) \mid \bar{\Psi}(t)\rangle=\left\langle\bar{\Psi}^{*}(t / 2) \mid \bar{\Psi}(t / 2)\right\rangle,
$$

where the last equality holds if the initial wave function is real and the Hamiltonian is symmetric. ${ }^{3}$ The last expression is preferable (and thus used in the following) because the wave function is more accurate at earlier times due to inexact numerical propagation and in the case of $\operatorname{MCTDH}[n]$ also the approximate form of $|\bar{\Psi}(t)\rangle$.

\section{B. Intramolecular vibrational-energy redistribution}

We first apply the $\operatorname{MCTDH}[n]$ methods to study the intramolecular vibrational-energy redistribution (IVR) of formyl fluoride-specifically the decay of the autocorrelation function. The initial wave packet is generated by performing a state-specific VSCF calculation on the one-mode part of the PES targeting the state where the $\mathrm{C}-\mathrm{H}$ stretch is excited to the second excited state $[0,0,0,0,0,2]$, i.e., an uncoupled-anharmonic-oscillator computation. The dynamics studied below is, thus, initiated from the coupling between modes. The MCTDH calculations are performed with $n^{m}=6 \forall m$. Figure 2 shows the absolute value of the autocorrelation function for $\operatorname{MCTDH}[n]$ with $n \in[2,4]$, using the different constraint operators. The results clearly show that three-mode excitations are necessary in order to describe the decay of the autocorrelation function. None of the MCTDH[2] methods reproduce the correct decay and optimizing the constraint operators does not improve the results significantly. For MCTDH[3], the MCTDH[3,V], and MCTDH[3,D] methods outperform MCTDH[3,g0] - especially after $10^{4}$ a.u. The MCTDH[4] wave functions include enough configurations to obtain high accuracy for all three choices of constraint.

\section{Accuracy of the MCTDH[n] wave functions}

We continue to examining the accuracy and convergence of the $\operatorname{MCTDH}[n]$ hierarchy for different choices of constraint operators. Figure 3 shows Hilbert-space angles and weights of the reference configuration for $\operatorname{MCTDH}[n]$ with $n \in[2,5]$ for a 6D HenonHeiles potential. The initial wave packets are generated by performing VSCF calculations on the harmonic part of the potential shifted by 0.5 in all directions. The results show clear convergence with respect to the excitation level-particularly for the $\operatorname{MCTDH}[n, \mathrm{D}]$ and $\operatorname{MCTDH}[n, \mathrm{~V}]$ methods. The low-order $\operatorname{MCTDH}[n, \mathrm{~g} 0]$ methods exhibit the largest errors and the effect of going from $n=2$ to $n=3$ is not impressive. This can be explained from the fact that the $\operatorname{MCTDH}[n, \mathrm{~g} 0]$ wave functions are not variational, and thus, it is not guaranteed that extending the parameter space leads to more accurate results. ${ }^{62}$ The $\mathrm{MCTDH}[n, \mathrm{~g} 0]$ EOMs do not at all take into account that the occupied modal in each mode is special, and therefore, the number of configurations needed for an accurate description of the dynamics is large compared to the two other methods. The $\mathrm{MCTDH}[n, \mathrm{D}]$ hierarchy converges nicely and the reference configurations have high weight during the entire propagation. The accuracy of the $\mathrm{MCTDH}[n, \mathrm{~V}]$ wave functions is comparable to $\operatorname{MCTDH}[n, \mathrm{D}]$. The density-matrix constraint is seen to provide more accurate wave functions for $n=2$ and $n=3$ at longer times,

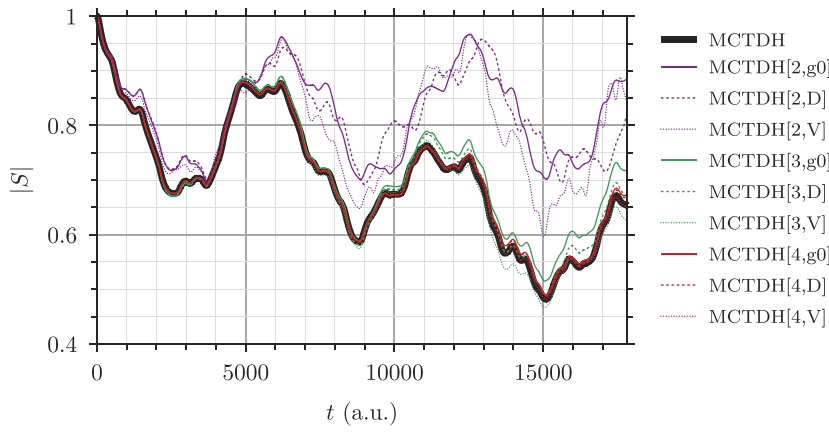

FIG. 2. Absolute value of the autocorrelation function of formyl fluoride, where the $\mathrm{C}-\mathrm{H}$ stretch is initially excited to the second excited state. For all methods, we use $n^{m}=6$ for all modes. 

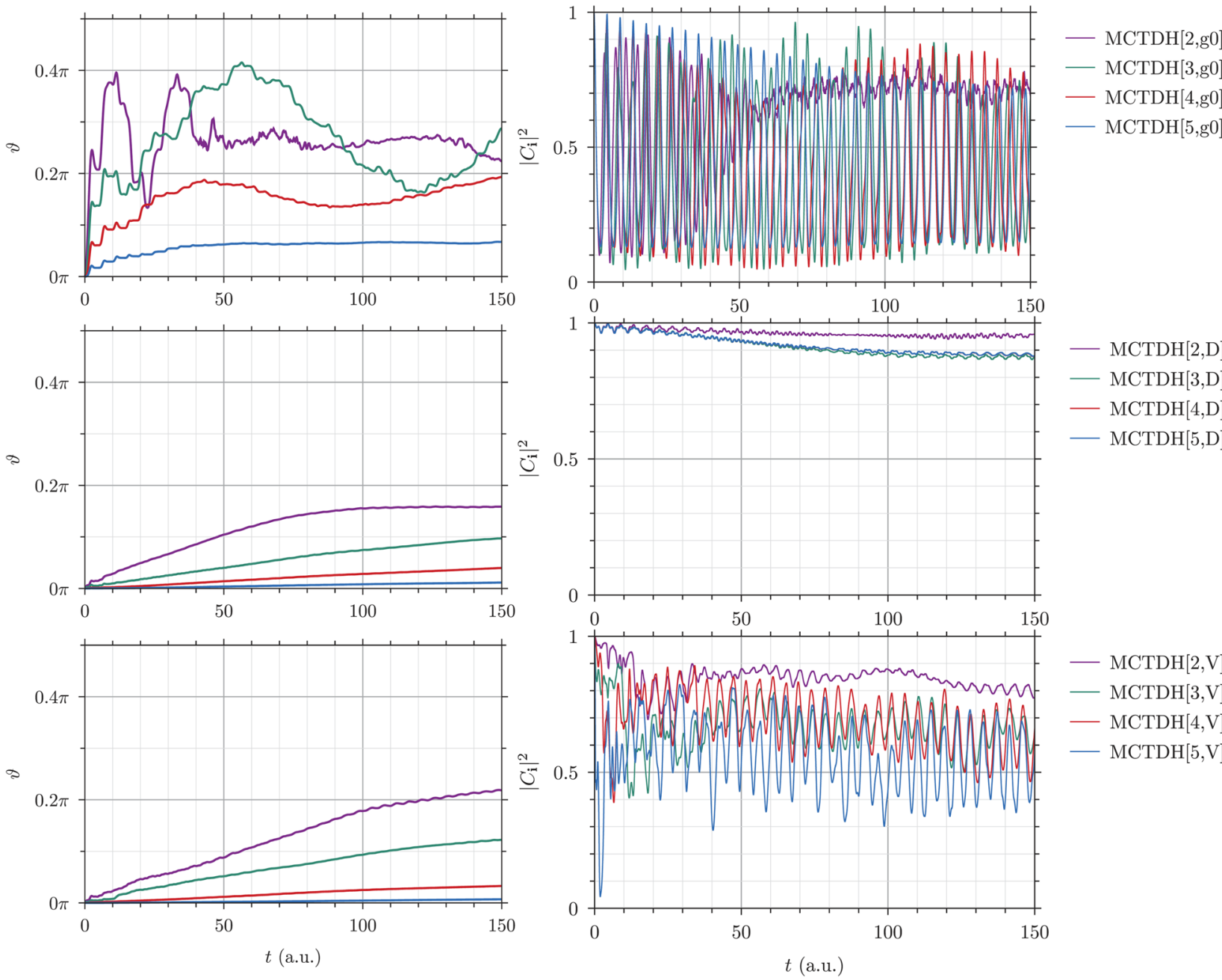

FIG. 3. Hilbert-space angles compared to the full MCTDH wave function (left column) and weight of the reference configuration of MCTDH[n] (right column) for a $6 \mathrm{D}$ HenonHeiles potential with $n^{m}=6 \forall \mathrm{m}$. Top: $g=0$, middle: natural-modal constraint, and bottom: variational constraint. The initial wave packet is a Gaussian shifted by 0.5 in all directions.

while the higher-order methods benefit most from the variational constraint. This may seem counterintuitive at first, but it is important to note that the TDVP only minimizes the local error of the time derivative. Thus, as also seen in Fig. 3, the short-time errors can be expected to be the smallest for the $\mathrm{MCTDH}[n, \mathrm{~V}]$ wave functions, while the behavior at longer times is less predictable.

In order to see if the same conclusions apply to a more complex system, we also present the Hilbert-space angles of the MCTDH $[n]$ wave functions for the IVR calculations on formyl fluoride presented in Sec. VI B. These are shown in Fig. 4. As for the 6D HenonHeiles potential, the $\operatorname{MCTDH}[n]$ hierarchy converges systematically toward the full MCTDH wave function. The MCTDH $[n, \mathrm{~V}]$ wave functions are most accurate at short times, but for $n=2$ and $n=3$, the picture becomes less clear later on. Overall, the accuracy does not depend strongly on the choice of the constraint operator, although



FIG. 4. Hilbert-space angles with respect to the full MCTDH wave function of the MCTDH[n] methods for the IVR calculations on formyl fluoride presented in Sec. VI B. 

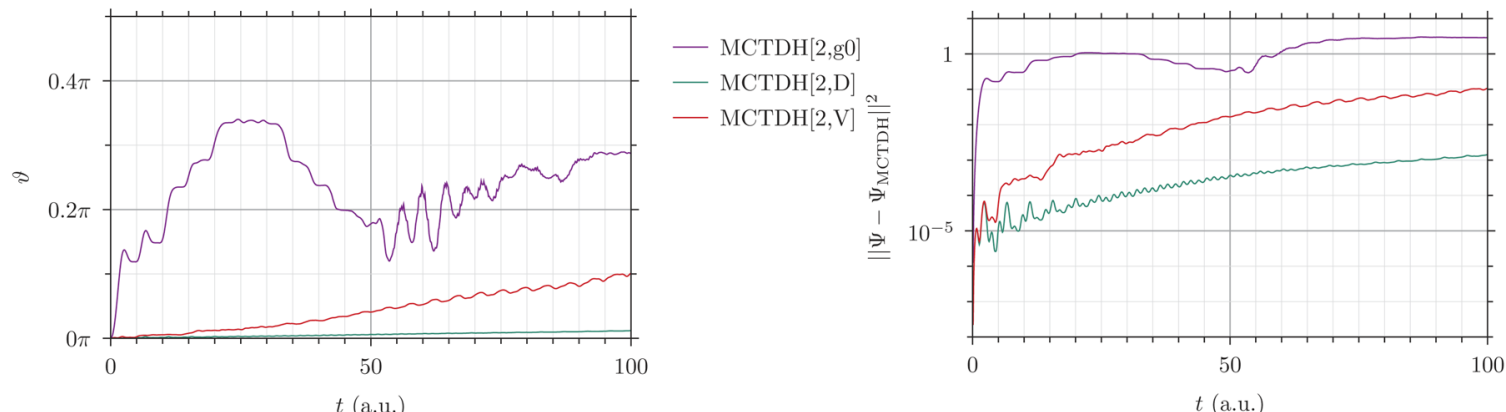

- $\mathrm{MCTDH}[2, \mathrm{~g} 0]$

- MCTDH $[2, \mathrm{D}]$

- MCTDH $[2, \mathrm{~V}]$
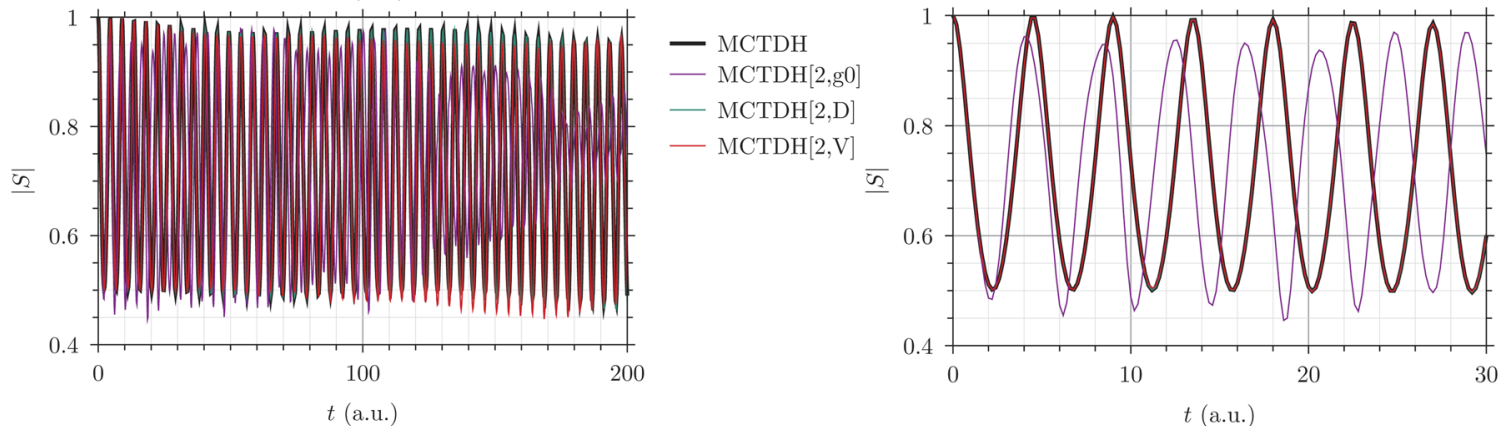

- MCTDH

- MCTDH[2,g0]

- MCTDH[2,D]

- MCTDH $[2, \mathrm{~V}]$

FIG. 5. Top: Hilbert-space angle and squared difference norm compared to the full MCTDH wave function for MCTDH[2] calculations on two uncoupled 2D Henon-Heiles potentials. Bottom: Autocorrelation function for full propagation and a zoom. The initial wave packet is a Gaussian shifted by 0.5 in all directions.
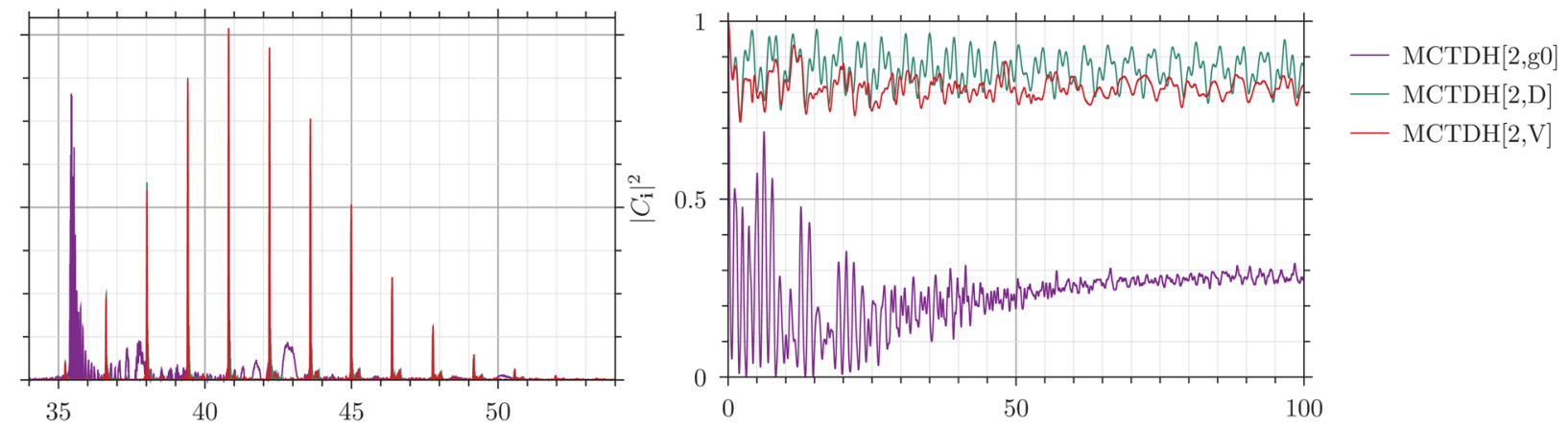

$\omega$ (a.u.)



35

40

45

50

$\omega$ (a.u.)

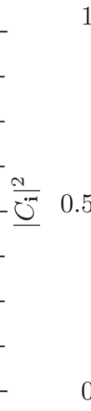

$0+$
0

$t$ (a.u.)

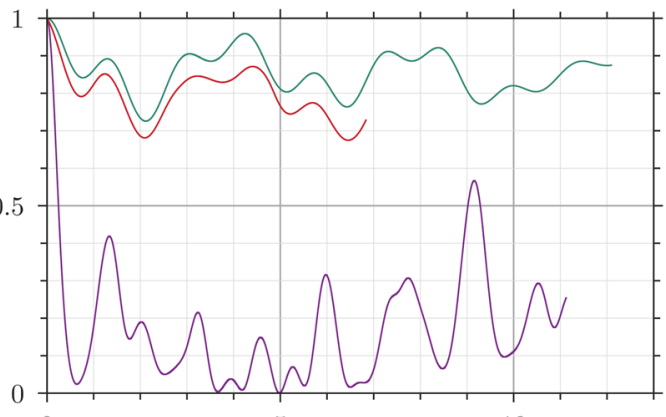

- $\mathrm{MCTDH}[3, \mathrm{~g} 0]$

- MCTDH[3,D]

- MCTDH[3,V]

FIG. 6. Spectra (left) and reference weights (right) for a 50D Henon-Heiles potential with $n^{m}=6 \forall m$. The initial wave packet is a Gaussian shifted by 0.5 in all directions. 



FIG. 7. Spectra (left) and reference weights (right) for a 100D Henon-Heiles potential with $n^{m}=6 \forall m$. The initial wave packet is a Gaussian shifted by 0.5 in all directions.

the MCTDH[4,V] wave function is the most accurate through the entire propagation.

\section{Separability}

We now study the separability of $\mathrm{MCTDH}[n]$ wave functions by applying the MCTDH[2] methods to a system of two uncoupled 2D Henon-Heiles potentials. For each of the two subsystems all MCTDH[2] wave functions are exact, but the time evolution of the modals matters in the description of the combined system where four-mode excitations are required for constructing the exact wave function. Figure 5 shows Hilbert-space angles, squared difference norms, and autocorrelation functions of the MCTDH[2] methods compared to the full MCTDH wave function. The results clearly show that the MCTDH[2,D] wave function performs much better than MCTDH[2,g0] because the weight of the reference configuration is kept large during the propagation as discussed in Sec. IV D. The MCTDH[2,V] wave function is also significantly better than $\mathrm{MCTDH}[2, \mathrm{~g} 0]$, but the density-matrix constraint still performs best because the reference weight is kept large instead of minimizing the importance of the three-mode excitations. None of the MCTDH[2] wave functions are exact (as opposed to the time-dependent VCC counterpart), but it is evident that the separability errors can in this case be kept small by optimizing the time evolution of the modals. We, however, expect the absolute errors to increase with the number of non-interacting subsystems and calculations performed on three uncoupled 2D Henon-Heiles potentials (not shown here) support this.

\section{E. High-dimensional Henon-Heiles potentials}

We now apply the $\operatorname{MCTDH}[n]$ methods to larger HenonHeiles potentials where full MCTDH calculations are unavailable. We note that the ML-MCTDH method has been applied to HenonHeiles systems with up to 1458 dimensions. ' However, in our present work, we choose the initial conditions such that all modes are displaced which will make the choice of a tree structure for MLMCTDH difficult, as the displaced modes cannot all belong to the same logical coordinate.

Figures 6-9 show data for 50D, 100D, 250D, and 500D HenonHeiles potentials, respectively. For all calculations, an active space of $n^{m}=6 \forall m$ is used, and the initial wave packet is generated by performing a VSCF calculation on a harmonic potential shifted by 0.5 in all modes resulting in a shifted Gaussian wave


$\operatorname{MCTDH}[2, \mathrm{~g} 0$
$-\operatorname{MCTDH}[2, \mathrm{D}]$

FIG. 8. Spectra (left) and reference weights (right) for a 250D Henon-Heiles potential with $n^{m}=6 \forall m$. The initial wave packet is a Gaussian shifted by 0.5 in all directions. 




FIG. 9. Absolute value of MCTDH[2] autocorrelation functions for a 500D HenonHeiles potential with $n^{m}=6 \forall m$. The initial wave packet is a Gaussian shifted by 0.5 in all directions.

packet. For the 50D potential, both MCTDH[2] and MCTDH[3] calculations are performed, while only MCTDH[2] is available for the larger systems. For the $500 \mathrm{D}$ potential, the auto-correlation function is shown instead of the spectrum because the spectral resolution is not as high as for the lower-dimensional systems. We note that especially for MCTDH[2,g0], the step sizes of the integrator became quite small compared to those of the corresponding $\mathrm{MCTDH}[2, \mathrm{D}]$ and MCTDH[2,V] calculations. This is the reason for the shorter propagation times for the $g=0$ constraint seen in Figs. 7-9.

The results clearly show that optimizing the constraint operators is key to obtaining physically meaningful autocorrelation functions and spectra. The MCTDH $[n, \mathrm{~g} 0]$ methods generally produce even qualitatively wrong wave functions which is due to the weights of the reference configuration dropping to $\sim 0$ very early. On the other hand, the $\operatorname{MCTDH}[n, \mathrm{D}]$ and $\operatorname{MCTDH}[n, \mathrm{~V}]$ methods are able to maintain a high weight of the reference configuration, and thus, calculate meaningful autocorrelation functions and spectra of these large systems. As seen in Fig. 6 the results obtained from $\mathrm{MCTDH}[2, \mathrm{~V}], \mathrm{MCTDH}[2, \mathrm{D}], \mathrm{MCTDH}[3, \mathrm{~V}]$, and $\mathrm{MCTDH}[3, \mathrm{D}]$ are very similar, and thus, including more configurations in the wave function is in this case less important than optimizing the time evolution of the modals. In other words, going from MCTDH[2,g0] to MCTDH[2,V] or MCTDH[2,D] improves the results much more than increasing the number of configurations by going to $\mathrm{MCTDH}[3, \mathrm{~g} 0]$.
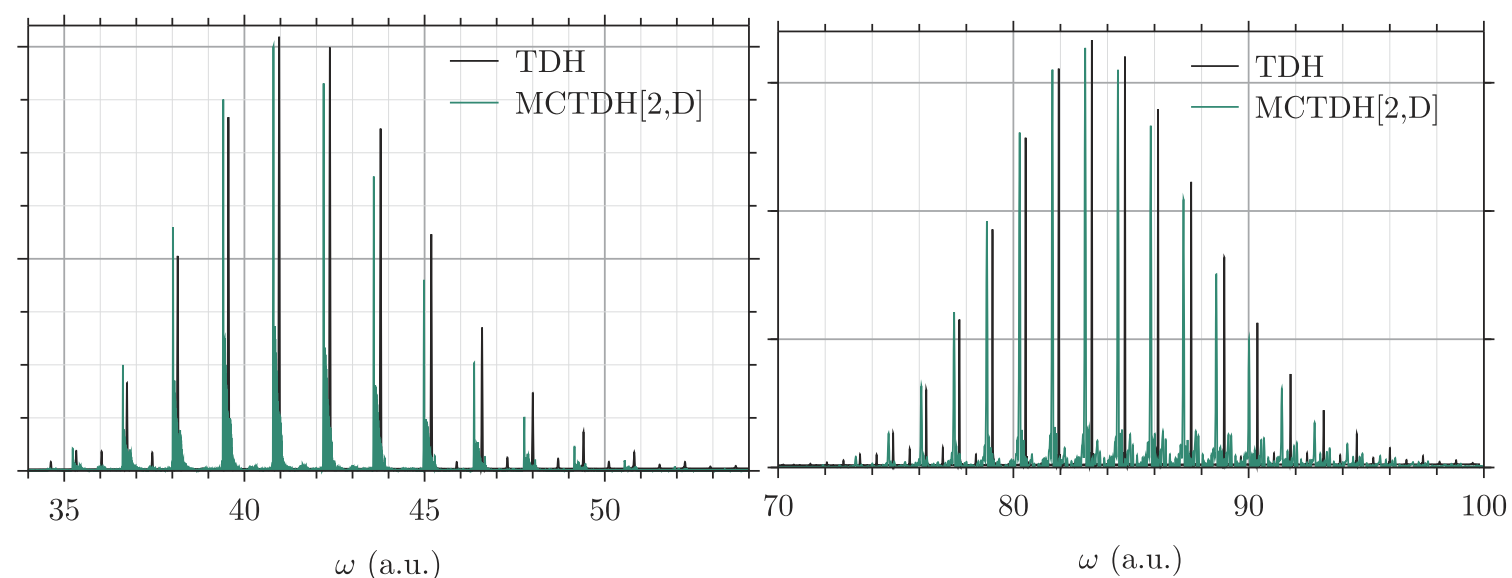

$\omega($ a.u. $)$

$\omega$ (a.u.)

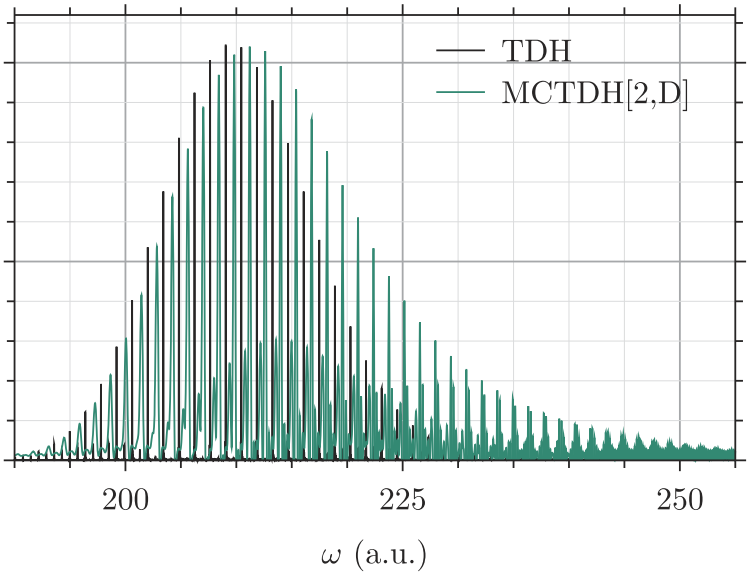

FIG. 10. Spectra for MCTDH[2,D] compared to X-TDH for 50D (top left), 100D (top right), and 250D (bottom) Henon-Heiles potentials. 
In Fig. 10, the MCTDH[2,D] results are compared to spectra obtained from $\mathrm{X}-\mathrm{TDH}^{4}$ calculations. The comparison with $\mathrm{TDH}$ spectra shows that the mean-field approximation is able to capture the low-energy structure of the spectra, but the complex patterns at high energy (especially seen in the 250D spectrum) are not reproduced at all in $\mathrm{TDH}$. We note that the TDH spectra resemble the MCTDH[2,D] spectra more than the MCTDH[2,g0] do. This can again be explained from the fact that $\mathrm{TDH}$ is a variational (and separable) theory, while MCTDH[2,g0] is not. Although the MCTDH[2,g0] wave function includes more configurations, the time evolution of the modals is not optimal which results in poor spectra.

\section{F. Timings and parallelization}

Table II shows central processing unit (CPU) times per derivative evaluation for formyl fluoride propagated to $t=100 \mathrm{a}$.u. using one thread on a CPU running at $2.3 \mathrm{GHz}$. The results show that significant computational savings are possible compared to using the full MCTDH method and that the number of configurations can be kept small, which reduces the memory requirements. It is important to note that the difference in the computational cost between MCTDH and a given MCTDH $[n]$ method will increase with the size of the system. Also note that the relatively high computational cost per derivative is due to the large number of terms in the formyl fluoride PES (2667).

We finally study the parallel speedup obtained by using sharedmemory parallelization (OpenMP) on the loop over operator terms. Figure 11 shows the parallel speedup (on a node with two 18-core CPUs running at $2.3 \mathrm{GHz}$ ) for formyl fluoride when integrating the MCTDH[3,D] EOMs for a time period of 100 a.u. 2.4 fs. The initial wave packet is obtained by performing a VSCF ground-state calculation on the one-mode part of the Hamiltonian. The speedup has been fitted to Amdahl's law,

$$
\operatorname{speedup}(N)=\frac{1}{(1-p)+\frac{p}{N}},
$$

where $N$ is the number of parallel processes. Thus, an estimate for the parallel proportion of the execution time, $p$, is obtained. The results show that a factor $\sim 16$ speedup is obtainable by using the parallel implementation on a node with 36 threads. The fit to Amdahl's law results in a parallel proportion of the execution time of $p=0.963$.

TABLE II. Number of configurations and CPU time per derivative evaluation (in seconds) for formyl fluoride.

\begin{tabular}{lrccc}
\hline \hline & & & \multicolumn{3}{c}{$t_{\text {deriv }}(\mathrm{s})$} \\
\cline { 3 - 5 } Excitation level & $N_{\text {config }}$ & $g 0$ & $D$ & $V$ \\
\hline 2 & 406 & 0.42 & 0.42 & 1.1 \\
3 & 2906 & 1.6 & 1.6 & 3.7 \\
4 & 12281 & 4.7 & 4.9 & 10 \\
Full & 46656 & 11 & & \\
\hline \hline
\end{tabular}

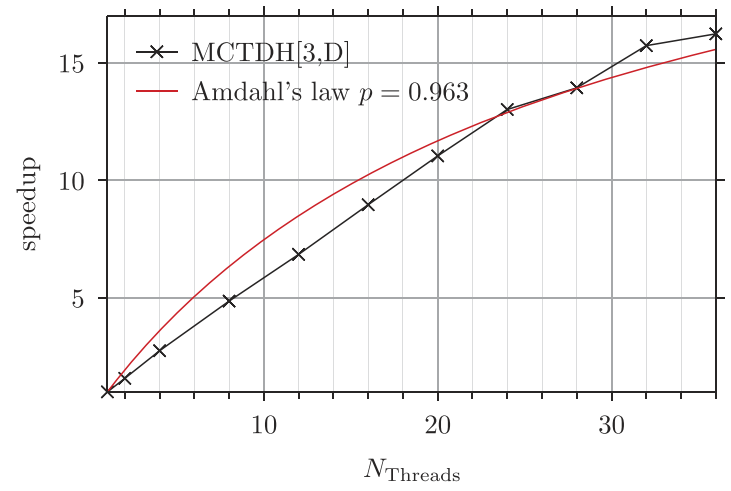

FIG. 11. Parallel speedup of an MCTDH[3,D] calculation on formyl fluoride with $n^{m}=6 \forall m$

\section{SUMMARY AND OUTLOOK}

The MCTDH EOMs have been derived in SQ and a new hierarchy of approximate methods, $\mathrm{MCTDH}[n]$, which reduce the number of configurations by including up to $n$-mode excitations with respect to a reference has been introduced. It has been shown that reducing the number of configurations removes some of the redundancies in the MCTDH wave function, which makes the $\mathrm{MCTDH}[n]$ wave functions depend on the choice of constraint (or gauge) operators, $g$. Linear equations for obtaining a fully variational $\operatorname{MCTDH}[n, \mathrm{~V}]$ wave function have been derived together with an alternative constraint, which keeps the one-mode density matrices block diagonal during the propagation resulting in the $\operatorname{MCTDH}[n, \mathrm{D}]$ methods. These have been compared to the $g=0$ case denoted as $\operatorname{MCTDH}[n, \mathrm{~g} 0]$.

The numerical results show that the $\operatorname{MCTDH}[n]$ hierarchy converges systematically toward the full MCTDH wave function and that optimizing the constraint operators is key to obtaining accurate results. The fully variational constraint leads to high accuracy, but the optimization of the $g^{m}$ operators makes it more expensive than the two other choices. Using the density-matrix constraint leads to cheaper propagation and physically meaningful results even in the case where $n$ is much smaller than the number of degrees of freedom in the system as exemplified by the MCTDH[2,D] calculations on Henon-Heiles potentials with up to 500 dimensions. The MCTDH $[n, \mathrm{D}]$ methods also perform well for non-interacting subsystems (compared to both $\mathrm{MCTDH}[n, \mathrm{~g} 0]$ and $\mathrm{MCTDH}[n, \mathrm{~V}]$ ) because the weight of the reference configuration is kept large during the propagation. However, the $\mathrm{MCTDH}[n]$ methods do by construction not behave correctly with respect to separability as opposed to the TDVCC method introduced in Ref. 26. We, thus, expect the TDVCC method to perform better for larger systems and the current investigation of constraint operators in $\mathrm{MCTDH}[n]$ is an important step on the way to introducing time-dependent modals in TDVCC.

The MCTDH $[n]$ methods introduced in this work truncate the configuration space based on a maximum excitation level alone. The MCR $[I]$ can, however, include selected higher-order excitations as long as it is closed under one-mode deexcitation for $\operatorname{MCTDH}[n, \mathrm{~V}]$. This is of importance when studying systems where 
one (or a few) degrees of freedom are special. An example could be double-well systems, but also when studying non-adiabatic dynamics using the single-set formalism ${ }^{3}$ a balanced description of the nuclear wave function on all PESs is required. An extension of $\operatorname{MCTDH}[n]$ to cover this case will be the topic of a forthcoming publication. As a further outlook, the MCR $[I]$ does not need to be constant in time. It is therefore possible to define criteria for dynamically including (or removing) higher-order excitations in the wave function during the propagation in line with Ref. 20.

Finally, an exponential parameterization of the modal transformation (possibly also the configuration space) in MCTDH inspired by the X-TDH method of Ref. 4 is also relevant for consideration. This non-linear parameterization will ensure that the active modals are kept orthonormal at all time eliminating the need for a nonorthogonal projector. Future research will tell if this leads to a more efficient implementation.

\section{SUPPLEMENTARY MATERIAL} material.

The PES for formyl fluoride is attached as the supplementary

\section{ACKNOWLEDGMENTS}

O.C. acknowledges support from the Danish Council for Independent Research through a Sapere Aude III Grant (No. DFF - 400200015), the Lundbeck Foundation, and the Danish e-infrastructure Cooperation (DeiC).

\section{REFERENCES}

${ }^{1}$ D. Tannor, Introduction to Quantum Mechanics: A Time-Dependent Perspective (University Science Books, Sausalito, California, USA, 2005).

${ }^{2}$ H.-D. Meyer, U. Manthe, and L. S. Cederbaum, Chem. Phys. Lett. 165(1), 73 (1990).

${ }^{3}$ M. Beck, A. Jackle, G. Worth, and H. Meyer, Phys. Rep. 324, 1 (2000).

${ }^{4}$ N. K. Madsen, M. B. Hansen, A. Zoccante, K. Monrad, M. B. Hansen, and O. Christiansen, J. Chem. Phys. 149(13), 134110 (2018)

${ }^{\mathbf{5}}$ N. Makri, Annu. Rev. Phys. Chem. 50(1), 167 (1999).

${ }^{6} \mathrm{H}$. Wang and M. Thoss, J. Chem. Phys. 119, 1289 (2003).

${ }^{7}$ H. Wang and M. Thoss, J. Chem. Phys. 131(2), 024114 (2009).

${ }^{8}$ U. Manthe, J. Chem. Phys. 128, 164116 (2008).

${ }^{9}$ O. Vendrell and H.-D. Meyer, J. Chem. Phys. 134, 044135 (2011).

${ }^{10}$ I. Burghardt, H. Meyer, and L. Cederbaum, J. Chem. Phys. 111, 2927 (1999).

${ }^{11}$ I. Burghardt, K. Giri, and G. A. Worth, J. Chem. Phys. 129(17), 174104 (2008).

${ }^{12}$ G. A. Worth and I. Burghardt, Chem. Phys. Lett. 368(3), 502 (2003).

${ }^{13}$ G. Richings, I. Polyak, K. Spinlove, G. Worth, I. Burghardt, and B. Lasorne, Int. Rev. Phys. Chem. 34(2), 269 (2015).

${ }^{14}$ D. V. Shalashilin and M. S. Child, J. Chem. Phys. 115(12), 5367 (2001).

${ }^{15}$ J. A. Green, A. Grigolo, M. Ronto, and D. V. Shalashilin, J. Chem. Phys. 144(2), 024111 (2016)

${ }^{16}$ B. F. E. Curchod and T. J. Martínez, Chem. Rev. 118(7), 3305 (2018).

${ }^{17}$ G. A. Worth, J. Chem. Phys. 112(19), 8322 (2000).

${ }^{18}$ R. Wodraszka and T. Carrington, J. Chem. Phys. 145(4), 044110 (2016).
${ }^{19}$ R. Wodraszka and T. Carrington, J. Chem. Phys. 146(19), 194105 (2017).

${ }^{20}$ H. R. Larsson and D. J. Tannor, J. Chem. Phys. 147(4), 044103 (2017).

${ }^{21}$ O. Christiansen, J. Chem. Phys. 120, 2140 (2004).

${ }^{22}$ O. Christiansen, Phys. Chem. Chem. Phys. 9(23), 2942 (2007).

${ }^{23}$ O. Christiansen, J. Chem. Phys. 120(5), 2149 (2004).

${ }^{24} \mathrm{P}$. Seidler and O. Christiansen, J. Chem. Phys. 131(23), 234109 (2009).

${ }^{25}$ N. K. Madsen, I. H. Godtliebsen, S. A. Losilla, and O. Christiansen, J. Chem. Phys. 148(2), 024103 (2018).

${ }^{26}$ M. B. Hansen, N. K. Madsen, A. Zoccante, and O. Christiansen, J. Chem. Phys. 151(15), 154116 (2019).

${ }^{27}$ O. E. Alon, A. I. Streltsov, and L. S. Cederbaum, J. Chem. Phys. 127(15), 154103 (2007).

${ }^{28}$ O. E. Alon, A. I. Streltsov, and L. S. Cederbaum, Phys. Rev. A 77(3), 033613 (2008).

${ }^{29}$ U. Manthe and T. Weike, J. Chem. Phys. 146(6), 064117 (2017).

${ }^{30} \mathrm{~T}$. Weike and U. Manthe, J. Chem. Phys. 152(3), 034101 (2020).

${ }^{31}$ H. Miyagi and L. B. Madsen, Phys. Rev. A 87(6), 062511 (2013).

${ }^{32}$ H. Miyagi and L. B. Madsen, Phys. Rev. A 89(6), 063416 (2014).

${ }^{33}$ D. J. Haxton and C. W. McCurdy, Phys. Rev. A 91(1), 012509 (2015).

${ }^{34}$ C. Lévêque and L. B. Madsen, New J. Phys. 19(4), 043007 (2017).

${ }^{35}$ M. Hansen, M. Sparta, P. Seidler, D. Toffoli, and O. Christiansen, J. Chem. Theory Comput. 6(1), 235 (2010).

${ }^{36}$ V. Nagalakshmi, V. Lakshminarayana, G. Sumithra, and M. D. Prasad, Chem. Phys. Lett. 217(3), 279 (1994).

${ }^{37} \mathrm{~T}$. Helgaker, P. Jørgensen, and J. Olsen, Molecular Electronic-Structure Theory (Wiley, 2000).

${ }^{38}$ H.-D. Meyer and H. Wang, J. Chem. Phys. 148(12), 124105 (2018).

${ }^{39}$ C. Lubich, Appl. Math. Res. Express 2015(2), 311.

${ }^{40}$ B. Kloss, I. Burghardt, and C. Lubich, J. Chem. Phys. 146(17), 174107 (2017).

${ }^{41}$ U. Manthe, J. Chem. Phys. 101(3), 2652 (1994).

${ }^{42}$ P.-O. Löwdin, Physi. Rev. 97(6), 1474 (1955).

${ }^{43}$ G. Battocchio, N. K. Madsen, and O. Christiansen, Mol. Phys. 115(1-2), 228 (2017).

${ }^{44}$ M. Beck and H.-D. Meyer, Z. Phys. D: At., Mol. Clusters 42(2), 113 (1997).

${ }^{45}$ O. Christiansen, D. Artiukhin, I. H. Godtliebsen, E. M. Gras, W. Győrffy, M. B. Hansen, M. B. Hansen, E. L. Klinting, J. Kongsted, C. König, S. A. Losilla, D. Madsen, N. K. Madsen, K. Monrad, G. Schmitz, P. Seidler, K. Sneskov, M. Sparta, B. Thomsen, D. Toffoli, and A. Zoccante, MidasCpp: Molecular Interactions, Dynamics and Simulation Chemistry Program Package in C++ (University of Aarhus, 2019), https://gitlab.com/midascpp/midascpp.

${ }^{46}$ M. Sparta, D. Toffoli, and O. Christiansen, Theor. Chem. Acc. 123(5-6), 413 (2009).

${ }^{47}$ E. L. Klinting, B. Thomsen, I. H. Godtliebsen, and O. Christiansen, J. Chem. Phys. 148(6), 064113 (2018).

${ }^{48}$ C. König and O. Christiansen, J. Chem. Phys. 145(6), 064105 (2016).

${ }^{49}$ B. Thomsen, K. Yagi, and O. Christiansen, Chem. Phys. Lett. 610-611, 288 (2014).

${ }^{50}$ E. L. Klinting, C. König, and O. Christiansen, J. Phys. Chem. A 119(44), 11007 (2015).

${ }^{51}$ J. M. Bowman, J. Chem. Phys. 68(2), 608 (1978).

${ }^{52}$ J. Bowman, Acc. Chem. Res. 19(7), 202 (1986).

${ }^{53}$ E. Hairer, G. Wanner, and S. P. Nørsett, Solving Ordinary Differential Equations I: Nonstiff Problems (Springer, 1993).

${ }^{54}$ P. Seidler, M. B. Hansen, and O. Christiansen, J. Chem. Phys. 128, 154113 (2008).

${ }^{\mathbf{5 5}}$ TURBOMOLE, a development of University of Karlsruhe and Forschungszentrum Karlsruhe GmbH, 1989-2007, TURBOMOLE GmbH, since 2007; available from http://www.turbomole.com. 
${ }^{56}$ C. Hättig, D. P. Tew, and A. Köhn, J. Chem. Phys. 132(23), 231102 (2010).

${ }^{57}$ K. A. Peterson, T. B. Adler, and H.-J. Werner, J. Chem. Phys. 128(8), 084102 (2008).

${ }^{58} \mathrm{~F}$. Weigend and M. Häser, Theor. Chem. Acc. 97(1), 331 (1997).
${ }^{\mathbf{5 9}}$ F. Weigend, M. Häser, H. Patzelt, and R. Ahlrichs, Chem. Phys. Lett. 294(1), 143 (1998).

${ }^{60}$ T. H. Dunning, J. Chem. Phys. 90(2), 1007 (1989).

${ }^{61}$ D. Toffoli, M. Sparta, and O. Christiansen, Mol. Phys. 109(5), 673 (2011).

${ }^{62}$ A. Raab, Chem. Phys. Lett. 319(5), 674 (2000). 